\title{
NET01 Regulates Postsynaptic Kainate Receptors in CA3 Interneurons During Circuit Maturation
}

\author{
Ester Orav ${ }^{1,2} \cdot$ Ilona Dowavic ${ }^{1,2} \cdot$ Johanna Huupponen ${ }^{1,2} \cdot$ Tomi Taira $^{2,3} \cdot$ Sari E. Lauri ${ }^{1,2}$ (D)
}

Received: 19 December 2018 / Accepted: 15 April 2019 /Published online: 1 May 2019

(C) The Author(s) 2019

\begin{abstract}
Kainate type ionotropic glutamate receptors (KARs) are expressed in hippocampal interneurons and regulate interneuron excitability and GABAergic transmission. Neuropilin tolloid-like proteins (NETO1 and NETO2) act as KAR auxiliary subunits; however, their significance for various functions of KARs in GABAergic interneurons is not fully understood. Here we show that NETO1, but not NETO2, is necessary for dendritic delivery of KAR subunits and, consequently, for formation of KAR-containing synapses in cultured GABAergic neurons. Accordingly, electrophysiological analysis of neonatal CA3 stratum radiatum interneurons revealed impaired postsynaptic and metabotropic KAR signaling in Neto1 knockouts, while a subpopulation of ionotropic KARs in the somatodendritic compartment remained functional. Loss of NETO1/KAR signaling had no significant effect on development of $\alpha$ amino-3-hydroxy-5-methyl-4-isoxazolepropionic acid (AMPA) and N-methyl-D-aspartate (NMDA)-receptor-mediated glutamatergic transmission in CA3 interneurons, contrasting the synaptogenic role proposed for KARs in principal cells. Furthermore, loss of NETO1 had no effect on excitability and characteristic spontaneous network bursts in the immature CA3 circuitry. However, we find that NETO1 is critical for kainate-dependent modulation of network bursts and GABAergic transmission in the hippocampus already during the first week of life. Our results provide the first description of NETO1-dependent subcellular targeting of KAR subunits in GABAergic neurons and indicate that endogenous NETO1 is required for formation of KAR-containing synapses in interneurons. Since aberrant KAR-mediated excitability is implicated in certain forms of epilepsy, NETO1 represents a potential therapeutic target for treatment of both adult and early life seizures.
\end{abstract}

Keywords Glutamate receptor - Kainate receptor · Neuropilin tolloid-like protein · Hippocampus · Circuit development · Auxiliary subunit

\section{Introduction}

Kainate type ionotropic glutamate receptors (KARs) modulate synaptic transmission and neuronal excitability in various parts of the brain and exhibit subunit, subcellular compartment, and cell type dependent functions [1-3]. Various combinations of GluK1-5 subunits form a functional KAR tetramer that may be complemented with neuropilin tolloid-like proteins (NETO1 and NETO2) that act as KAR auxiliary

Sari E. Lauri

sari.lauri@helsinki.fi

1 Molecular and Integrative Biosciences Research Program, University of Helsinki, PO Box 65, Viikinkaari 1, 00014 Helsinki, Finland

2 HiLife Neuroscience Center, University of Helsinki, Helsinki, Finland

3 Department of Veterinary Biosciences, University of Helsinki, Helsinki, Finland subunits $[1,3]$. NETOs provide an additional level of regulation to KAR localization and function in neurons [4]. For instance, at the glutamatergic CA3-MF synapse in the hippocampus, NETO1 regulates synaptic targeting, biophysical properties, and ligand affinity of postsynaptic GluK2/3containing KARs [5-7].

KARs are expressed in hippocampal interneurons and, when activated, have robust effects on GABAergic transmission [1-3, 8, 9]. Based on pharmacological studies, genetic mouse models, and mRNA expression pattern, interneuronal KARs in the hippocampus contain subunits GluK1, GluK2, or both [7, 10-15]. Of the GluK1 c-terminal splice variants, GluK1b is specifically expressed in interneurons while GluK1c is restricted to principal cells and mainly expressed during early development [16]. While KARs mediate a prominent inward current in response to agonist application, the synaptic KAR-mediated excitatory postsynaptic current (EPSC), described in CA1 interneurons, is rather modest $[10,17,18]$. Activation of KARs increases interneuron firing 
by membrane depolarization that consequently leads to higher GABAergic drive onto principal cells. In addition, axonal and presynaptic KARs regulate GABA release, likely via both ionotropic and metabotopic $G$ protein coupled signaling [1-3, 8, 9]. At immature CA3 stratum lucidum interneurons, tonically activated KARs have a developmentally restricted $G$ protein coupled function that regulates interneuron excitability by inhibiting medium after hyperpolarizing current $I_{\mathrm{mAHP}}$ [19]. Thus, hippocampal interneurons contain distinct subpopulations of KARs that regulate interneuron excitability and GABAergic drive both in the immature and in the adult hippocampi.

Numerous studies have documented NETO/KAR functional interactions in the principal neurons in adult [20] and recently also in the newborn hippocampus [21]; however, much less is known about NETO involvement in regulating KARs in interneurons. Neto1 is expressed in GABAergic interneurons in the adult hippocampus $[5,22]$ where it regulates agonist-induced KAR currents and KAR-dependent recruitment of inhibitory drive onto principal cells [22].

Here, we have further studied the role of NETO1/KAR interaction in hippocampal interneurons, focusing on its physiological role during the first 2 weeks of postnatal development. We show that NETO1, but not NETO2, is necessary for dendritic delivery of KAR subunits and, consequently, for formation of KAR containing synapses in GABAergic neurons. In contrast to principal neurons where KARs promote formation and maturation of glutamatergic synapses [21, 23-26], loss of NETO1/KAR signaling had no significant effects on development of AMPAR-containing synapses in GABAergic interneurons in culture or in area CA3 of the hippocampus. Moreover, NETO1 was not indispensable for maintenance of basal network excitability in the immature hippocampus but was necessary for kainate-induced modulation of GABAergic transmission and network bursts.

\section{Methods}

Animals Male and female wild-type (WT), Netol knockout (KO), and Neto2KO (C57Bl/6NCrl) mice $[6,27]$ were used in this study. The animal experiments were performed in accordance with the University of Helsinki Animal Welfare Guidelines.

Cell Culture and Lentiviral Infection Primary hippocampal dispersed neuron culture was prepared from P0-2 old WT, Neto1KO, and Neto2KO mice pups as previously described [21]. Cultured neurons were infected with lentiviruses to express GFP, GluK1b-flag, GluK1c-flag, and GluK2-myc at days in vitro 3 (DIV3) and fixed at DIV14 using 4\% PFA $4 \%$ Sucrose in PBS. Lentiviral plasmids and production of virus particles used in this study were described earlier by Vesikansa and colleagues in 2012 [16].

Immunofluorescence Fixed neurons were permeabilized with $0.2 \%$ Triton X-100 and incubated with PBS-based blocking solution containing $5 \%$ goat serum, $2 \%$ bovine serum albumin (BSA), $0.1 \%$ Triton X-100, and $0.05 \%$ Tween-20. Primary antibodies (Table 1) were diluted in the blocking solution and incubated overnight at $+4{ }^{\circ} \mathrm{C}$. Secondary antibodies (Table 2) were diluted in PBS and incubated $1 \mathrm{~h}$ at room temperature.

The stained samples were mounted on microscope slides using Prolong Gold antifade reagent (P36934, Life Technologies). Confocal images were acquired using a LSM Zeiss 710 confocal microscope (alpha PlanApochromat $63 \times / 1.46$ OilKorr M27 objective) for KAR dendritic targeting experiments. All other samples were imaged using Leica TCS SP8 confocal microscope and HC PL APO $93 \times / 1.30$ motCORR STED WHITE (glycerol) and $3 \times$ digital zoom to obtain high resolution images for synapse analysis.

Image Analysis All samples were blinded for genotype and KAR subunit expression during staining, image acquisition, and analysis. Samples included in the analysis were obtained from 2 to 3 independent culture batches. $n$ number represents the total number of neurons analyzed. GAD67 staining was used in all samples to identify GAD67+ putative interneurons. Under our culture conditions, $9.7 \% \pm 1.0 \%$ of cultured neurons was positive for GAD67 $(n=333)$. Dendritic and axonal targeting of overexpressed taggedKARs was analyzed in MAP2 positive dendrites and MAP2 negative axons of GAD67+ neurons using SynD/ MATLAB [28] by measuring flag or myc intensity along the neurite and normalizing it to soma intensity. Synaptic clusters were visualized using co-staining against presynaptic (Synaptophysin, red) and postsynaptic (PSD95, green) markers. Synaptic cluster density was analyzed from 3D construction using Imaris software. The Synaptophysin (Syn) and PSD95 puncta included in the analysis had spot diameter of at least $0.5 \mu \mathrm{m}$ on $X Y$-axis and $1 \mu \mathrm{m}$ on $Z$-axis. Syn and PSD95 spots within a distance of $0.7 \mu \mathrm{m}$ were considered as synaptic clusters. In some samples, KAR and/or GluA2/4-containing synapses were analyzed. Similarly, minimum KAR or GluA2/4 spot diameter on $X Y$-axis was $0.5 \mu \mathrm{m}$ and on $Z$-axis $1 \mu \mathrm{m}$. KAR and GluA2/4 spots were considered to co-localize if the distance between the spots were max $0.35 \mu \mathrm{m}$. KAR spot was considered to be extrasynaptic if the distance between the flag/ myc spot and PSD95 spot was above $0.35 \mu \mathrm{m}$. All spot size measurements were calculated from the center of the spot using Imaris software. Syn, PSD95, KAR, and GluA2/4 spots, and synaptic clusters were confirmed visually. 
Table 1 Primary antibodies used in this study

\begin{tabular}{llll}
\hline Antibody & Dilution & Product nr & Manufacturer \\
\hline Guinea pig anti-Synaptophysin & $1: 2000$ & 101,004 & Synaptic Systems \\
Mouse anti-PSD95 & $1: 1000$ & $75-028$ & NeuroMab \\
Chicken anti-GAD67 & $1: 2000$ & 198,006 & Synaptic Systems \\
Mouse anti-GAD67 & $1: 1000$ & MAB5406 & Millipore \\
Rabbit anti-GAD67 & $1: 2000$ & 198,013 & Synaptic Systems \\
Rabbit anti-flag & $1: 1000$ & F7425 & Sigma Aldrich \\
Rabbit anti-myc & $1: 1000$ & $06-549$ & Millipore \\
Chicken anti-MAP2 & $1: 8000$ & AB5543 & Millipore \\
Mouse anti-GluA2/4 & $1: 2500$ & MAB396 & Millipore \\
\hline
\end{tabular}

Acute Slice Preparation Acute parasagittal hippocampal sections $(350 \mu \mathrm{m})$ were prepared from brains of WT and Neto1KO mice using dissection solution containing (in $\mathrm{mM}$ ): $87 \mathrm{NaCl}, 2.5 \mathrm{KCl}, 7 \mathrm{MgCl}_{2}, 1.25 \mathrm{NaH}_{2} \mathrm{PO}_{4}, 0.5$ $\mathrm{CaCl}_{2}, 25 \mathrm{NaHCO}_{3}, 50 \mathrm{D}$-sucrose, and $25 \mathrm{D}$-glucose and equilibrated with $95 \% \mathrm{O}_{2}$ and $5 \% \mathrm{CO}_{2}$. Slices were transferred to artificial cerebrospinal fluid (ACSF) containing (in $\mathrm{mM}): 124 \mathrm{NaCl}, 3 \mathrm{KCl}, 1.25 \mathrm{NaH}_{2} \mathrm{PO}_{4}, 3 \mathrm{MgSO}_{4}, 26$ $\mathrm{NaHCO}_{3}, 2 \mathrm{CaCl}_{2}$, and 15 D-glucose and incubated $30 \mathrm{~min}$ at $+35^{\circ} \mathrm{C}$ and at $30 \mathrm{~min}-4 \mathrm{~h}$ at room temperature before use.

Electrophysiological Recordings Whole-cell voltage clamp recordings were performed from CA3 stratum radiatum interneurons that were visually identified with differential interference contrast (DIC) optics. No further identification of interneuron subtype was undertaken.

During the recordings, the chamber was continuously perfused with ACSF $\left(32{ }^{\circ} \mathrm{C}\right)$ bubbled with $95 \% \mathrm{O}_{2}$ and $5 \% \mathrm{CO}_{2}$. AMPAR-KAR-mediated responses were recorded in the presence of $100 \mu \mathrm{M}$ picrotoxin (Abcam) and $50 \mu \mathrm{M}$ D-AP5 (HelloBio) at a holding potential $-70 \mathrm{mV}$. NMDARmediated responses were recorded at a holding potential + $40 \mathrm{mV}$ and in the presence of $10 \mu \mathrm{M} \mathrm{CNQX} \mathrm{(Abcam)} \mathrm{and}$ $100 \mu \mathrm{M}$ picrotoxin. One micromolar of tetrodotoxin (TTX, Abcam) was added to the drug cocktail for recording of miniature excitatory postsynaptic currents (mEPSC). In order to isolate KAR component of the evoked response, AMPAR- selective antagonist GYKI53655 $(30 \mu \mathrm{M})$ was added to the bath solution. In some experiments, GluK1-specific antagonist ACET (200 nM, Tocris) and agonist ATPA ( $1 \mu \mathrm{M}$, Tocris $)$ were used.

Glutamatergic currents were recorded with 3-5 M $\Omega$ glass electrodes filled with Cs-based intracellular solution containing (in mM): $130 \mathrm{CsMeSO}_{4}, 10$ HEPES, 0.5 EGTA, 4 Mg-ATP, $0.3 \mathrm{Na}$-GTP, 5 QX-314, $8 \mathrm{NaCl}$, and 285 mOsm ( $\mathrm{pH}$ 7.2). For evoked EPSC, the stimulation electrode was placed in CA3 stratum radiatum. For recording of after hyperpolarizing current $\left(I_{\mathrm{mAHP}}\right)$, the filling solution contained (in $\mathrm{mM}$ ): $130 \mathrm{~K}$-gluconate, 10 HEPES, $10 \mathrm{KCl}, 4$ ATP-Mg, 0.3 GTP-Mg, 0.2 EGTA, and $285 \mathrm{mOsm}$ (pH 7.2). $I_{\mathrm{mAHP}}$ was induced by applying depolarizing $60 \mathrm{mV} 40 \mathrm{~ms}$ step from holding potential of $-47 \mathrm{mV}$. Spontaneous network activity was recorded from CA3 pyramidal neurons voltage-clamped at $47 \mathrm{mV}$ with pipette filling solution containing (in $\mathrm{mM}$ ): $135 \mathrm{~K}$-gluconate, 10 HEPES, $2 \mathrm{KCl}, 2 \mathrm{Ca}(\mathrm{OH})_{2}, 5$ EGTA, $4 \mathrm{Mg}$-ATP, and 0.5 Na-GTP. Spontaneous action potential firing was measured in cell-attached configuration using $10 \mathrm{M} \Omega$ electrodes filled with ACSF.

Data were collected using Axoscope 9.2 (Axon instruments) and WinLTP software [29]. For whole-cell patch clamp recordings uncompensated series resistance $\left(R_{\mathrm{S}}<\right.$ $30 \mathrm{M} \Omega$ ) was monitored, and cells were discarded if $R_{\mathrm{s}}$ varied more than $20 \%$.
Table 2 Secondary antibodies used in this study

\begin{tabular}{llll}
\hline Antibody & Dilution & Product nr & Manufacturer \\
\hline Goat anti-chicken Alexa Fluor 405 & $1: 2000$ & ab175674 & Abcam \\
Goat anti-mouse Alexa Fluor 488 & $1: 2000$ & A11029 & Life Technologies \\
Goat anti-guinea pig Alexa Fluor 568 & $1: 2000$ & A11075 & Life Technologies \\
Goat anti-rabbit Alexa Fluor 647 & $1: 2000$ & A-21245 & Molecular Probes \\
Goat anti-mouse Alexa Fluor 647 & $1: 2000$ & A21236 & Life Technologies \\
\hline
\end{tabular}


Spontaneous events were analyzed with MiniAnalysis 6.0.3 program (Synaptosoft Inc.) and calculated in 1 min bins. Events were verified visually, and events with amplitude less than three times the baseline noise level were rejected. Evoked EPSC and $I_{\mathrm{mAHP}}$ amplitude was analyzed using WinLTP. Holding current data were collected during $I_{\mathrm{mAHP}}$ recordings.

Statistical Analysis All statistical analysis was performed on raw data using SigmaPlot software. First, the data distribution was tested with Shapiro-Wilk test. Then, one-way ANOVA with Holm-Sidak post hoc comparison or Kruskal-Wallis test was used accordingly as stated. Student's paired $t$ test was used to assess the treatment effect as indicated. All data are presented as mean $\pm \mathrm{SEM} ; p<0.05$ was considered statistically significant. In figures, the significance levels are indicated by asterisks as follows: $* p<0.05, * * p<0.01$, and $* * * p<0.001$.

\section{Results}

\section{NET01 Regulates Dendritic Targeting but Not Synaptic Recruitment of KAR Subunits GluK1b and GluK2 in GAD67+ GABAergic Neurons In Vitro}

NETO1/2 has been suggested to promote plasma membrane entry and synaptic targeting of KAR subunits depending on the cell type and KAR subunit identity [4, 20]. However, currently, there is no consensus on the precise mechanisms that guide the subcellular compartmentalization of KARs at GABAergic interneurons. Therefore, we characterized the subcellular localization of recombinant tagged KAR subunits in cultured GAD67 positive neurons from wild-type (WT), NETO1- and NETO2-deficient mice, focusing on the subunits GluK1 and GluK2. Both the GluK1b and GluK1c splice variants were included in the analysis.

All the GluK subunits studied were targeted to MAP2 positive dendrites and MAP2 negative axons in WT GAD67+ neurons. The relative intensity of GluK2 was higher as compared to GluK1b and GluK1c in both dendrites (GluK2 0.71 \pm 0.04 ; GluK1b $0.41 \pm 0.01, p<0.001$, Kruskal-Wallis; and GluK1c $0.40 \pm 0.01, p<0.001$, Kruskal-Wallis) and axons (GluK2 $0.38 \pm 0.02$; GluK1b $0.21 \pm 0.01, p<0.001$, Kruskal-Wallis; and GluK1c $0.21 \pm 0.01, p<0.001$, Kruskal-Wallis) of GAD67+ neurons. Dendritic delivery of all three subunits was significantly lower in the dendrites of GAD67+ Neto1KO neurons as compared to controls (GluK1b $75.3 \% \pm 2.2 \%, p<0.001$, Kruskal-Wallis; GluK1c $74.6 \% \pm$ $2.6 \%, p<0.001$, Kruskal-Wallis; GluK2 $79.9 \% \pm 3.5 \%, p=$ 0.01 , Kruskal-Wallis) (Fig. 1a, c). Also, axonal delivery of the KAR subunits was significantly lower in the GAD67+ Neto1KO neurons than controls (GluK1b 80.6\% $\pm 3.80 \%$, $p=0.001$; GluK1c $86.0 \% \pm 4.1 \%, p=0.031$, Kruskal-
Wallis; GluK2 79.7\% $\pm 4.0 \%, p=0.004$ ) (Fig. 1b, d). In contrast, we found no differences in dendritic (GluK1b 101.6\% \pm $6.2 \%, p=0.82$; GluK1c $101.3 \% \pm 4.6 \%, p=0.69$; GluK2 $109.5 \% \pm 3.4 \%, p=0.072$ ) (Fig. 1a, c) or axonal (GluK1b $110.0 \% \pm 7.5 \%, p=0.26$; GluK1c $112.3 \% \pm 8.5 \%, p=0.11$; GluK2 $105.7 \% \pm 5.9 \%, p=0.49$ ) (Fig. 1b, d) delivery of the tested KAR subunits in Neto2KO neurons as compared to WT controls. Therefore, we excluded Neto2KO from further study.

We then analyzed the distribution of the KAR subunits between synaptic and extrasynaptic pools, using puncta with co-localized Synaptophysin (Syn) and PSD95 staining as a synapse marker. At WT GAD67+ neurons, only a minority of the recombinant GluK subunits co-localized with SynPSD95 positive puncta (GluK1b $12.0 \% \pm 1.4 \%$, GluK1c $13.1 \% \pm 1.0 \%$, GluK2 $12.5 \% \pm 2.2 \%$ ). This distribution was not significantly altered in Neto1KO for the subunits GluK1b and GluK2 (GluK1b 13.6\% $\pm 1.4 \%$; GluK2 11.5\% $\pm 1.2 \%$ ). However, proportion of synaptically located GluK1c in Neto1KO GABAergic neurons $(6.4 \% \pm 1.1 \%)$ was significantly lower as compared to WTs $(p<0.001)$ (Fig. 2a, b).

Thus, under our culture conditions, subcellular localization of KAR subunits in GABAergic neurons depends on endogenous expression of NETO1 but not NETO2. Our findings further suggest that NETO1 regulates dendritic targeting rather than synaptic recruitment of interneuron specific KAR subunits GluK1b and GluK2. In contrast, synaptic localization of the GluK1c splice variant, which is endogenously expressed in the hippocampal pyramidal neurons during early development [16], was dependent on NETO1 expression.

\section{NET01 Is Required for Postsynaptic and Metabotropic KAR Functions in Immature CA3 Interneurons}

Interneurons in area CA3 of hippocampus express NETO1 already during the first week of life [21]. To understand the physiological functions of NETO1/KAR complex in the immature GABAergic neurons, we performed electrophysiological recordings in CA3 stratum radiatum interneurons in acute hippocampal slices from neonatal WT and Neto1KO mice.

Postsynaptic KARs contribute to synaptic transmission at certain interneurons $[10,17,30]$; however, it is not known whether KAR-mediated EPSCs at GABAergic neurons are modulated by NETO1. To investigate this, we placed a stimulation electrode to CA3 stratum radiatum in order to activate all possible glutamatergic inputs to the recorded cell and used pharmacological tools to isolate putative KAR-mediated postsynaptic currents. At P5 WT slices, application of the AMPAR-selective antagonist GYKI53655 $(30 \mu \mathrm{M})$ in the presence of antagonists for NMDAR and $\mathrm{GABA}_{\mathrm{A}}$ receptors revealed a small slowly decaying EPSC in response to 5 pulse $50 \mathrm{~Hz}$ afferent stimulation (peak amplitude $9.4 \pm 1.1 \mathrm{pA}$ ) (Fig. 3a). This current component is likely mediated by 
a
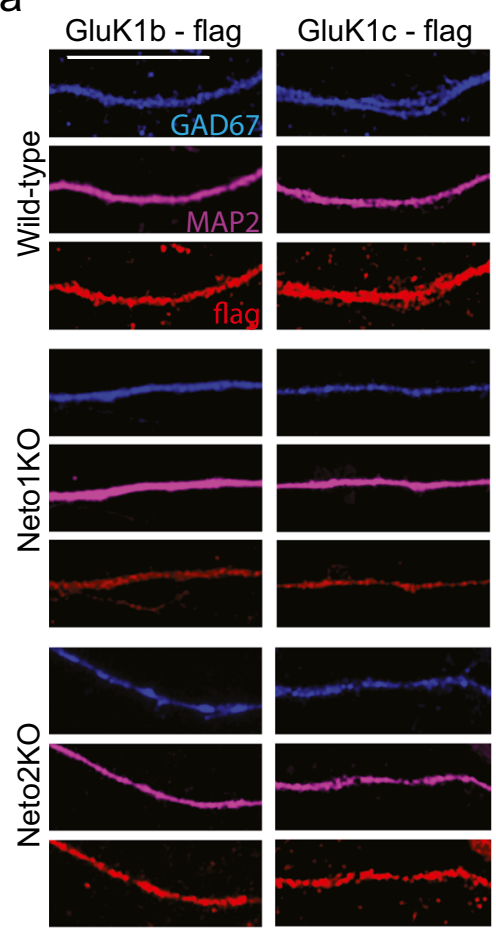

C

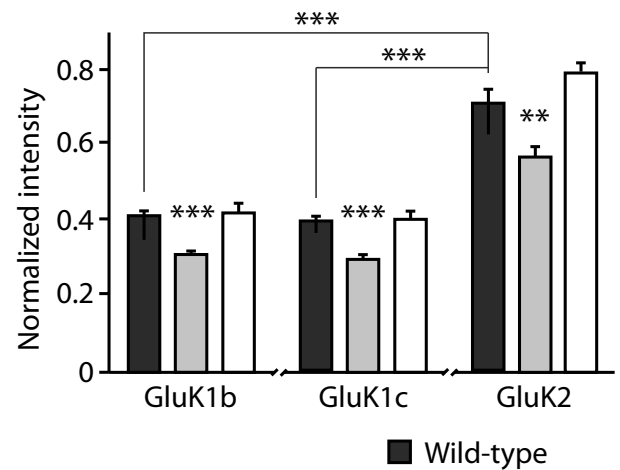

Fig 1 NETO1 regulates dendritic and axonal delivery of KAR subunits in cultured GAD67+ GABAergic neurons. a Example images depicting the localization of overexpressed GluK1b-flag, GluK1c-flag and GluK2-myc (red) in dendrites of cultured WT, Neto1KO and Neto2KO GABAergic neurons. MAP2 (purple) is used as dendritic marker, while GAD67 staining (blue) identifies GABAergic neurons. Scale bar $20 \mu \mathrm{m}$. b Examples illustrating the localization of overexpressed GluK1b-flag, GluK1c-flag, and GluK2-myc (red) in MAP2-negative axons of GABAergic neurons in the same cultures as in a. Scale bar $20 \mu \mathrm{m}$. c Quantification of the

synaptic KARs as it was significantly reduced with AMPARKAR antagonist CNQX ( $50 \mu \mathrm{M}$; EPSC amplitude to $65.5 \% \pm$ $4.8 \%$ of control, $p=0.001$, Student's $t$ test) (Fig. 3a).

Having detected functional synaptic KARs in immature CA3 interneurons in WTs, we then performed the same experiment using Neto1KO slices. Application of GYKI53655 completely blocked the EPSCs in response to 5 pulse $50 \mathrm{~Hz}$ stimulation in Neto1KO slices (peak amplitude 5.1 $\pm 0.4 \mathrm{pA}$ ), and application of CNQX had no further effect on the response b

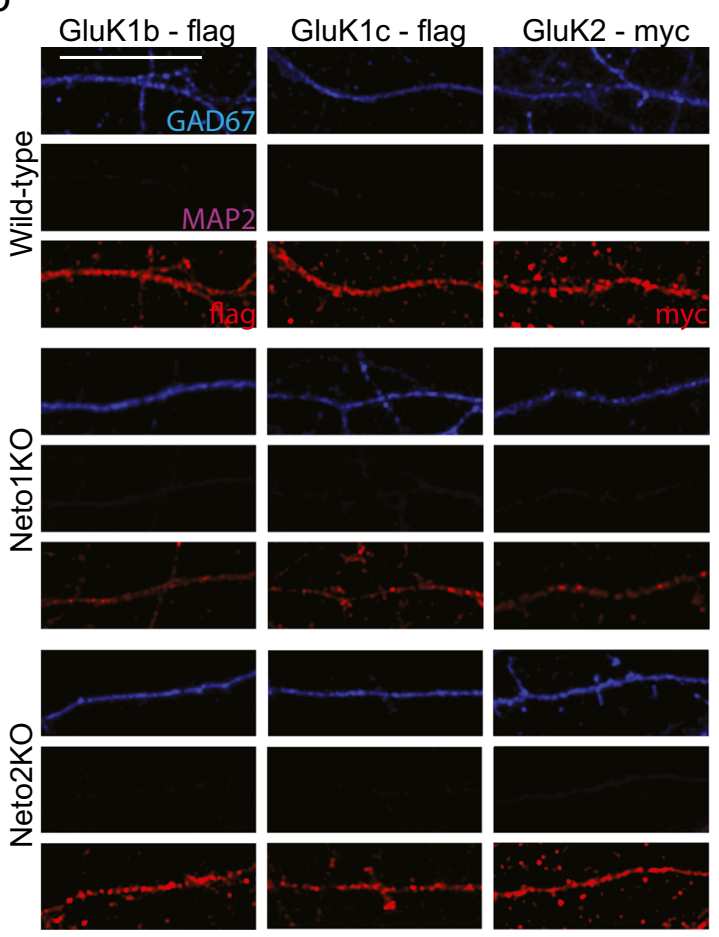

d

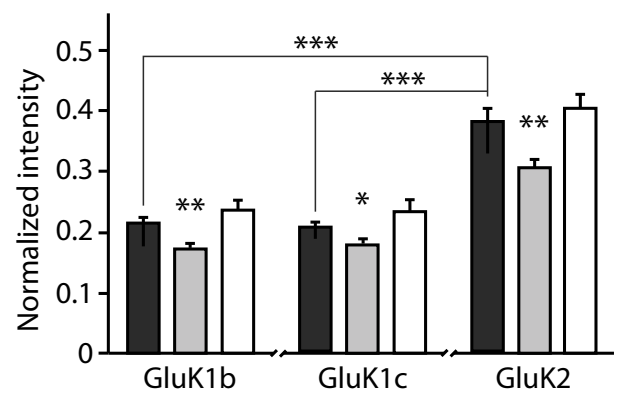

Neto1KO

Neto2KO

dendritic targeting of KAR subunits GluK1b ( $n=49, n=34, n=18)$, GluK1c $(n=49, n=33, n=15)$, and GluK2 $(n=42, n=32, n=18)$ in WT (black bar), Neto1KO (gray bar), and Neto2KO (white bar) GAD67+ neurons, respectively. d Quantification of the axonal targeting of KAR subunits GluK1b $(n=40, n=34, n=12)$, GluK1c $(n=45, n=33, n=12)$, and GluK2 ( $n=23, n=26, n=18)$ in WT (black bar), Neto1KO (gray bar), and Neto2KO (white bar) GAD67+ neurons, respectively. For quantification, GluK signal intensity in the neurites is normalized to the soma intensity. $* p<0.05 ; * * p<0.01 ; * * * p<0.001$

$(92.8 \% \pm 7.5 \%$ of control) (Fig. 3a). These data indicate that ionotropic KARs are present at the glutamatergic synapses inCA3 stratum radiatum interneurons, where they mediate a modest EPSC that is not observed in the absence of NETO1.

In addition to the ionotropic function, KARs in CA3 stratum lucidum interneurons activate a $\mathrm{G}$ protein coupled signaling pathway that inhibits medium after hyperpolarizing potassium current $\left(I_{\mathrm{mAHP}}\right)$ during the first week of postnatal development [19]. To investigate the possible role of NETO1 in the 
a

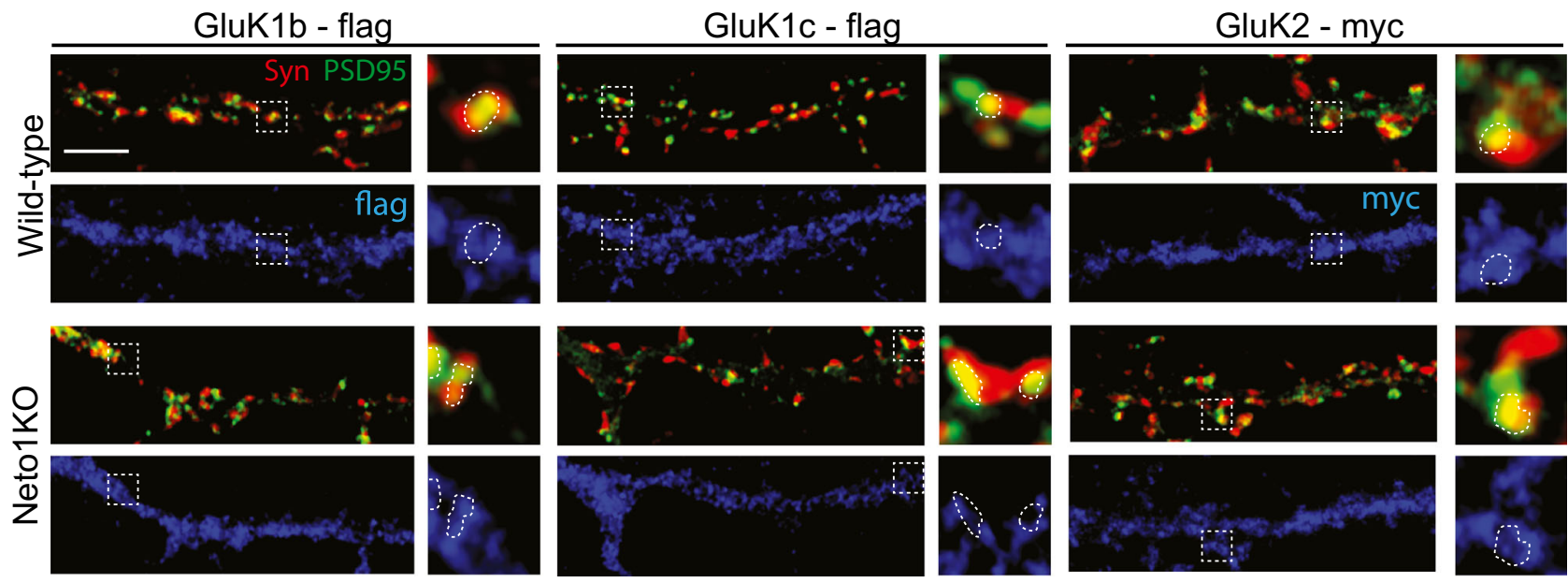

b

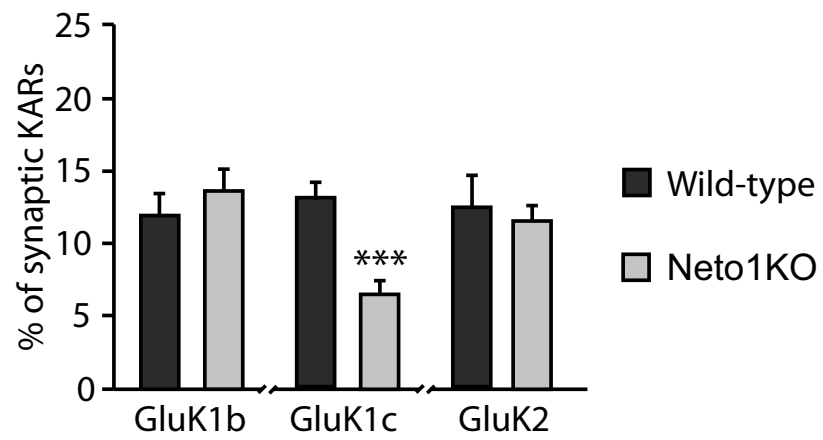

Fig. 2 Synaptic localization of KAR subunits in cultured WT and Neto1KO GABAergic neurons. a Example images of immunostaining against Synaptophysin (Syn, red), PSD95 (green), and tagged KAR subunits (flag/myc, blue) in dendrites of WT and Neto1KO GAD67+ neurons. Enlarged insets of a single synapse cluster (co-localization of Syn and PSD95, circled with a dashed line) and corresponding synaptically and extrasynaptically located GluK1b-flag, GluK1c-flag, and

metabotropic signaling initiated by GluK1-containing KARs, we studied the effect of GluK1-selective agonist ATPA $(1 \mu \mathrm{M})$ on $I_{\mathrm{mAHP}}$ in neonatal (P4-6) WT and Neto1KO slices. The $I_{\mathrm{mAHP}}$ amplitude was not different between the genotypes under basal conditions (WT 137.0 \pm 19.8 pA, Neto1KO 139.3 $\pm 14.5 \mathrm{pA})$. ATPA had no significant effect on WT $I_{\mathrm{mAHP}}$ currents when all recorded cells were included in the analysis $(97.7 \% \pm 3.5 \%$ of control; $n=12)$. However, ATPA significantly decreased $I_{\mathrm{mAHP}}$ amplitude in 5 out of the $12 \mathrm{WT}$ cells $(87.4 \% \pm 0.4 \%$ of control, $p=0.03$, Student's $t$ test). In contrast, ATPA did not reduce $I_{\mathrm{mAHP}}$ in any of the 10 recorded CA3 interneurons in Neto1KO $(104.7 \% \pm 2.9 \%$ of control) (Fig. $3 b)$. It should be noted that our recordings included interneurons located in CA3 stratum radiatum, while the previous study was restricted to interneurons in CA3 stratum lucidum [19], probably explaining why the effect of ATPA was observed only in a subpopulation of the neurons.

During these recordings, ATPA induced an inward current in WT interneurons, detected as an increase in
GluK2-myc clusters. GAD67 staining not shown for clarity. Scale bar $20 \mu \mathrm{m}$. b Pooled data showing percent of synaptically located KARs in WT (black; GluK1b-flag $n=16$, GluK1c-flag $n=17$, GluK2-myc $n=20$ ) and Neto1KO (gray; GluK1b-flag $n=20$, GluK1c-flag $n=10$, GluK2myc $n=20$ ) GAD67+ neurons. Scale bar $20 \mu \mathrm{m} . * p<0.05$; $* * p<0.01$; $* * * p<0.001$

the holding current during the whole-cell voltage clamp recording $(\Delta I 39.3 \pm 8.9 \mathrm{pA})$. Interestingly, a clear ATPA-induced current was also detected in Neto1KO interneurons $(\Delta I 12.3 \pm 2.1 \mathrm{pA})$; however, it was significantly smaller as compared to WT ( $p=0.007$, KruskalWallis) (Fig. 3c).

Taken together, these findings show that NETO1 is required for many, but not all, of the functions described for KARs in immature GABAergic interneurons. Loss of NETO1 impaired postsynaptic and metabotropic KAR signaling, while a subpopulation of ionotropic GluK1-containing KARs remained functional.

\section{Absence of NET01 Selectively Impairs Formation of KAR-Containing Synapses in GAD67+ GABAergic neurons}

Both ionotropic and metabotropic KAR signaling have been implicated in synaptogenesis and synapse maturation in 
a

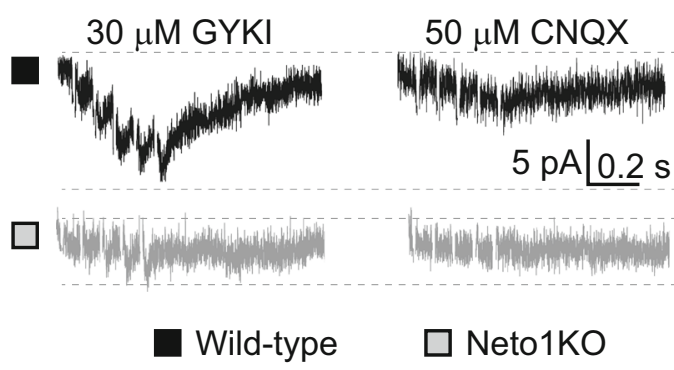

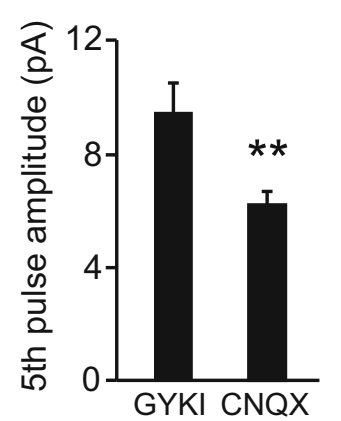

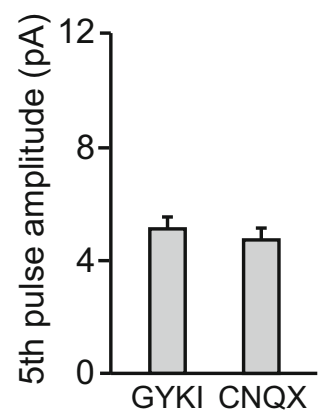

b
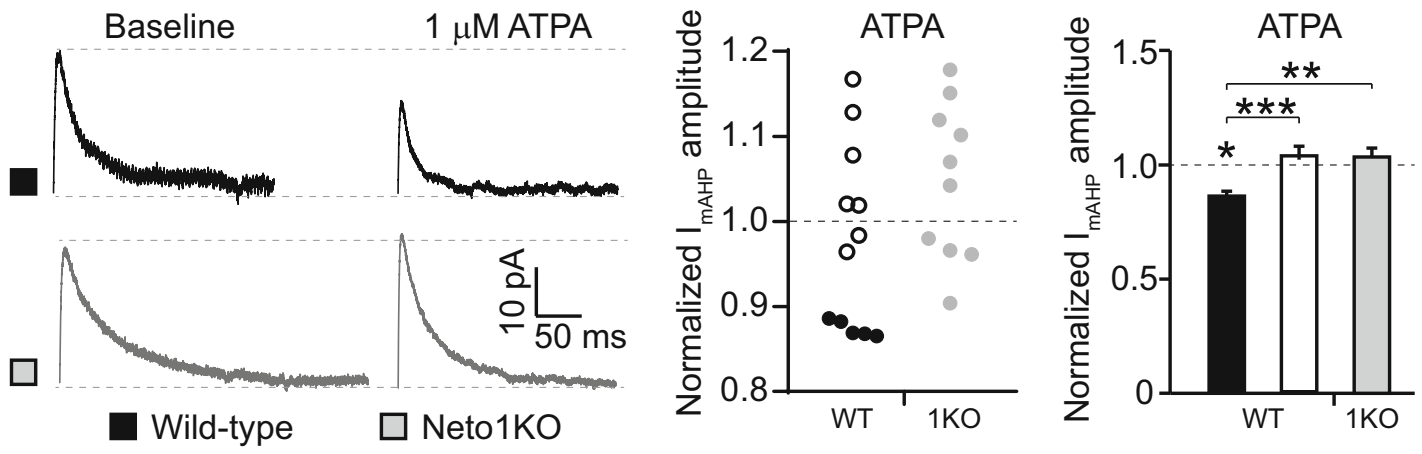

C
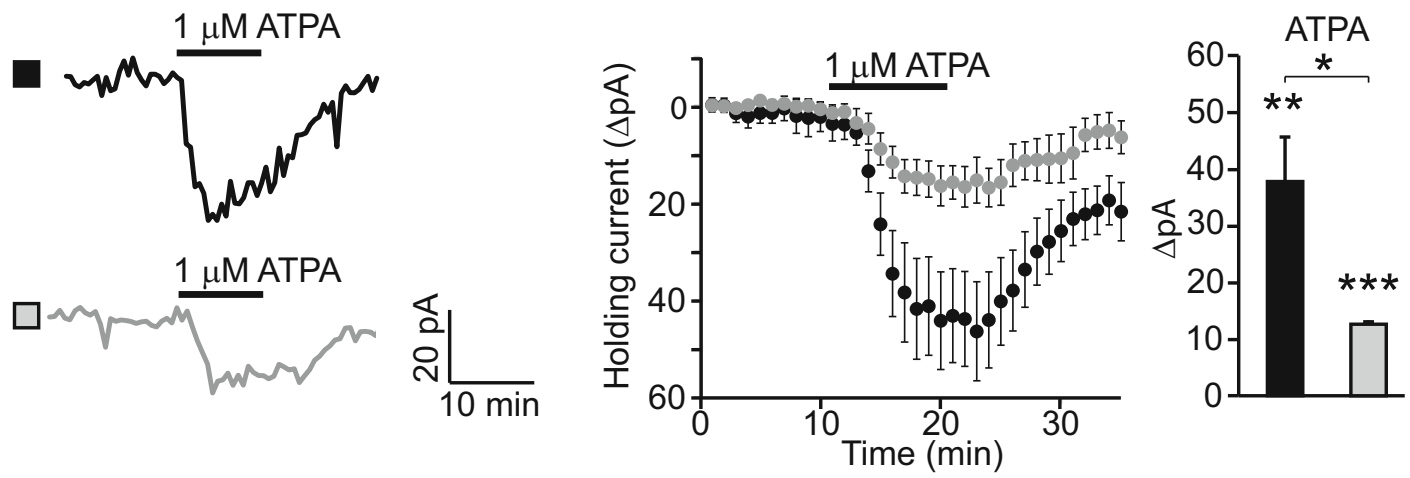

Wild-type

Fig. 3 NETO1 regulates postsynaptic and metabotropic KAR functions in neonatal CA3 stratum radiatum interneurons. a Example traces and pooled data demonstrating the effect of $30 \mu \mathrm{M}$ GYKI and $50 \mu \mathrm{M}$ CNQX on EPSC, evoked by 5 pulse at $50 \mathrm{~Hz}$ stimulation of mixed afferents, in WT $(n=10)$ and Neto1KO $(n=7) \mathrm{CA} 3$ stratum radiatum interneurons at P4-6. b Example traces and quantified data illustrating the effect of ATPA $(1 \mu \mathrm{M})$ on $I_{\mathrm{mAHP}}$ currents. Pooled data show the effect of ATPA on $I_{\mathrm{mAHP}}$

hippocampal principal neurons $[16,23,31]$. Therefore, we next investigated the role of NETO1/KAR complex in the formation of glutamatergic synapses in cultured GAD67+ neurons from WT and Neto1-null mice.

The density of synapses, identified as puncta with colocalized PSD95 and Synaptophysin (Syn) staining, was significantly lower in Neto1KO GAD67+ neurons $(0.42 \pm 0.02 /$ $\mu \mathrm{m})$ as compared to WT $(0.53 \pm 0.03 / \mu \mathrm{m}, p=0.01)$ (Fig. 4a).

This phenotype was KAR-dependent as it was fully rescued with the overexpression of either GluK1b $(0.51 \pm 0.04 / \mu \mathrm{m})$ or GluK2 $(0.49 \pm 0.01 / \mu \mathrm{m})$, but not GluK1c $(0.39 \pm 0.04 / \mu \mathrm{m}$, $p=0.04$, as compared to WT) in Neto1 KO cultures (Fig. 4a).

To further characterize this phenotype, we analyzed the density of KAR and AMPAR-containing synapses in KARoverexpressing GAD67+ neurons. AMPAR synapses were defined as co-localized clusters of staining against 
a
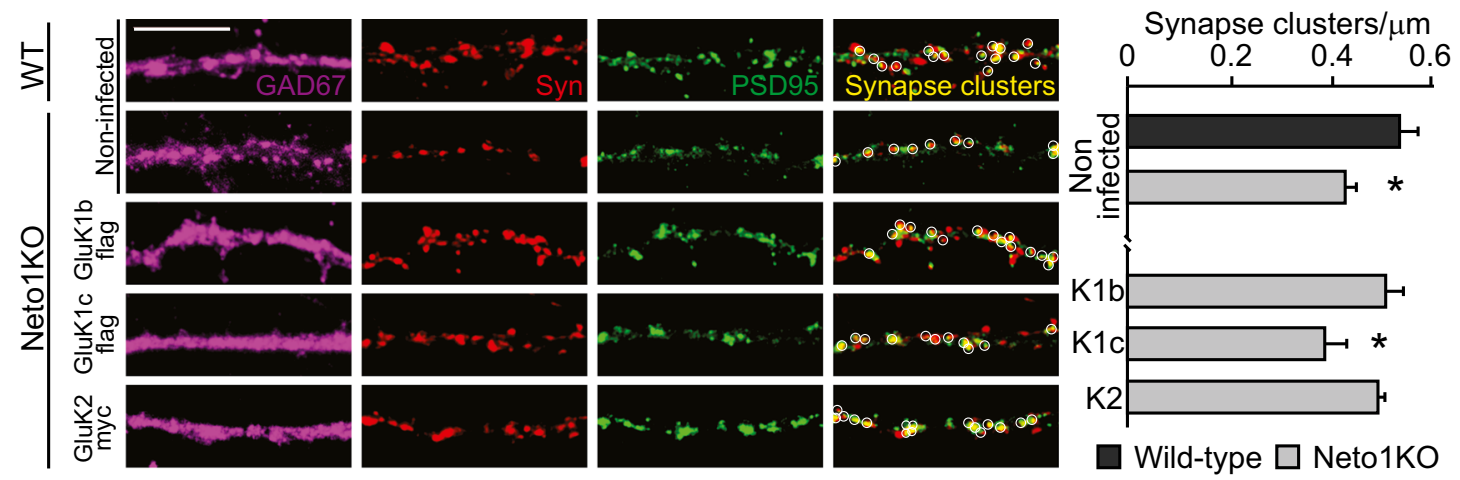

b

Wild-type

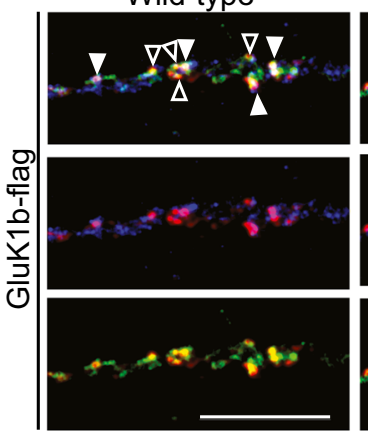

Neto1KO

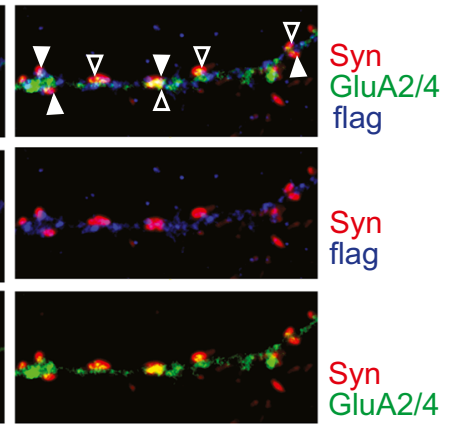

C
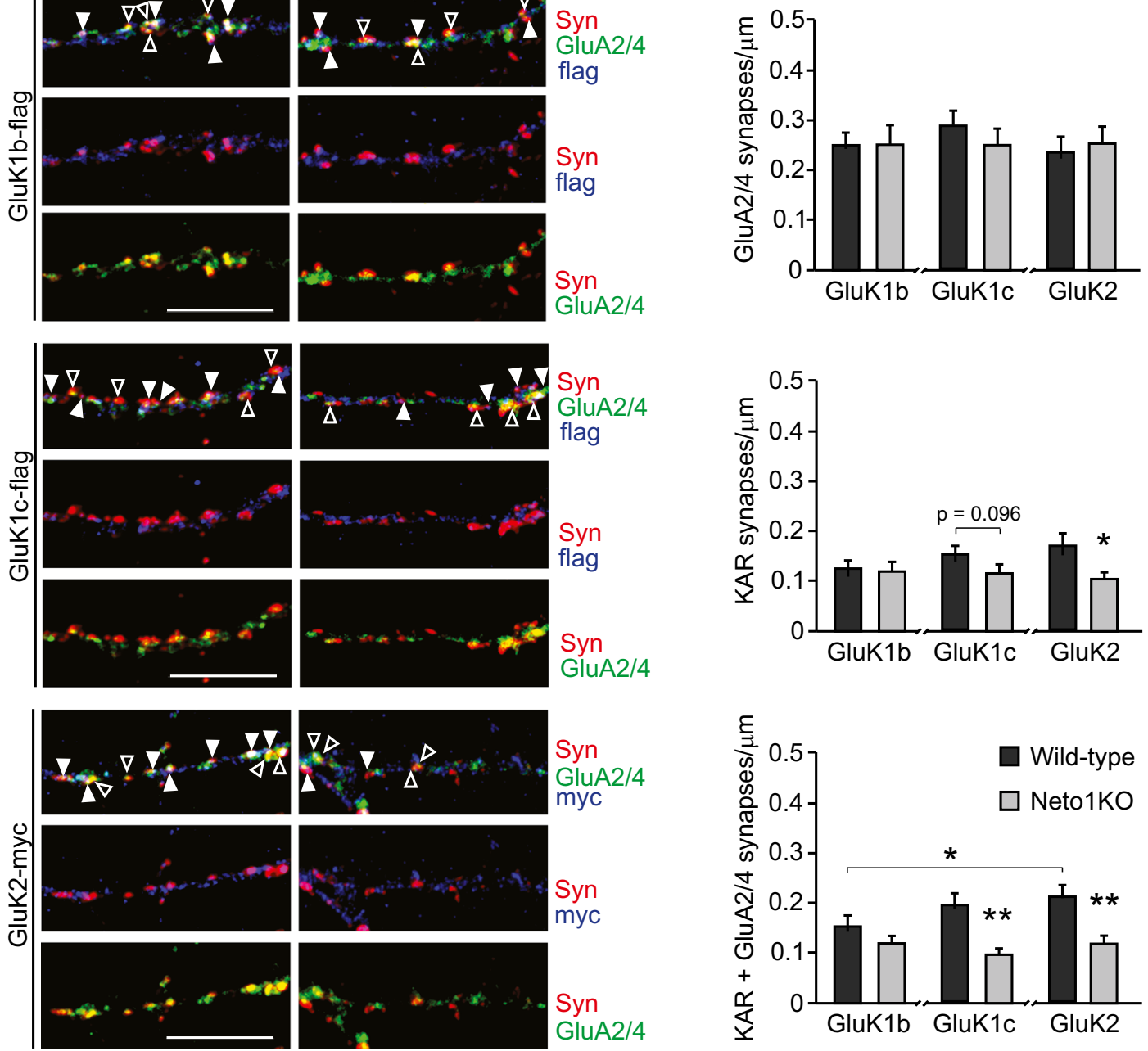

Syn

GluA2/4

myc
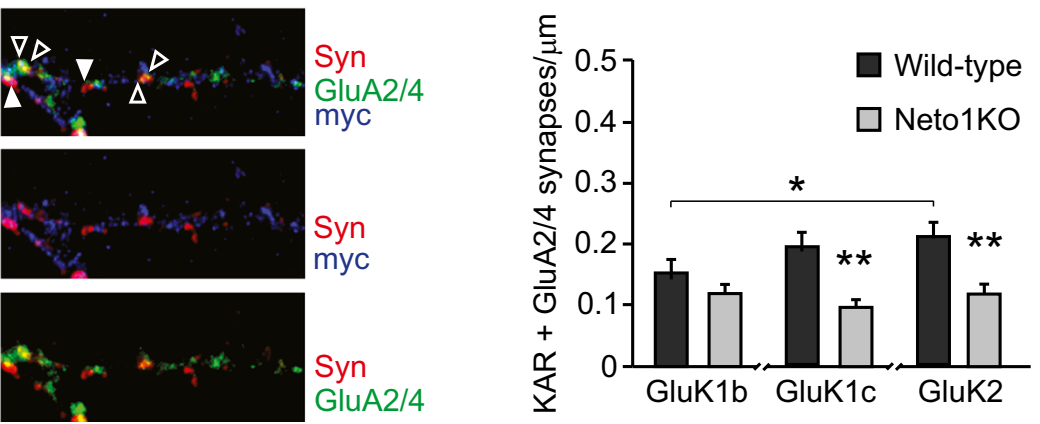

Fig. 4 Loss of NETO1 leads to selective impairment of KAR-containing synapses in cultured GAD67+ GABAergic neurons. a Example images showing immunostaining against GAD67 (purple), Synaptophysin (Syn, red) and PSD95 (green) in hippocampal neurons. Merged images show synapses (co-localization of Syn and PSD95, circled) in non-infected WT $(n=35)$ and Neto1KO $(n=30)$, and in GluK1b-flag $(n=20)$, GluK1cflag $(n=10)$ and GluK2-myc $(n=20)$ overexpressing Neto1KO GAD67+ neurons. Graphs show the quantified synapse density for the corresponding experimental conditions. Scale bar $10 \mu \mathrm{m}$. b Example images of Synaptophysin (Syn, red); GluA2/4 (green); and GluK1b-flag,

GluK1c-flag, or GluK2-myc (blue) staining in WT and Neto1KO GAD67+ neurons. Synapses are identified as having a contact with Synaptophysin and highlighted with arrowheads: GluA2/4 positive (open arrowhead), KAR positive (closed arrowhead), or KAR and GluA2/4 positive (closed arrowhead). GAD67+ staining not shown for clarity. Scale bar $10 \mu \mathrm{m}$. c Quantified data for the density of GluA2/4 positive, KAR positive, or KAR and GluA2/4 positive synapses in WT and Neto1KO GAD67+ neurons. GluK1b-flag (WT $n=20$, Neto1KO $n=$ 20), GluK1c-flag (WT $n=20$, Neto1KO $n=18$ ), or GluK2-myc (WT $n=19$, Neto1KO $n=20) . * p<0.05 ; * * p<0.01 ; * * * p<0.001$ 
endogenous GluA2/4 and Syn and represented $44 \%$ of the glutamatergic synapses in the WT GAD67+ neurons. KAR synapses were visualized as clusters of overexpressed tagged KAR subunits that co-localized with Syn (25\% of synapses). The remaining $31 \%$ of glutamatergic synapses contained both AMPARs (GluA2/4) and KARs.

The density of synapses that contained only AMPARs in GAD67+ neurons was not different between WT and Neto1KO cultures, irrespective of the overexpressed KAR subunit (GluK1b $0.25 \pm 0.02 / \mu \mathrm{m}, 0.25 \pm 0.04 / \mu \mathrm{m}$; GluK1c $0.29 \pm$ $0.03 / \mu \mathrm{m} ;$ GluK2 0.23 $\pm 0.03 / \mu \mathrm{m}, 0.25 \pm 0.03 / \mu \mathrm{m}$; WT and Neto1KO, respectively) (Fig. 4b, c; open arrowhead). In contrast, the density of KAR-only synapses was significantly more sparse in Neto1KO as compared to WTs in GluK2-expressing $(0.17 \pm 0.02 / \mu \mathrm{m}$ and $0.10 \pm 0.01 / \mu \mathrm{m}, p=0.02$, Kruskal-Wallis, WT and Neto1KO, respectively), but not in GluK1expressing GAD67+ GABAergicneurons (GluK1b $0.12 \pm$ $0.02 / \mu \mathrm{m}$ and $0.12 \pm 0.02 / \mu \mathrm{m}$; GluK1c $0.15 \pm 0.02 / \mu \mathrm{m}$ and $0.11 \pm 0.02 / \mu \mathrm{m}, \mathrm{WT}$ and Neto1KO, respectively) (Fig. 4b, c; closed arrowhead). Similarly, the density of synapses containing both KARs and AMPARs was affected by loss of NETO1 as evidenced by lower density of GluK1c + GluA2/4 or GluK2 + GluA2/4-containing synapses in Neto1KO as compared to WT controls (GluK1c 0.19 $\pm 0.02 / \mu \mathrm{m}$ and $0.09 \pm 0.01 / \mu \mathrm{m}, p=$ 0.001 , Kruskal-Wallis; GluK2 $0.21 \pm 0.02 / \mu \mathrm{m}$ and $0.11 \pm$ $0.02 / \mu \mathrm{m}, p=0.02$, Kruskal-Wallis, WT and Neto1KO, respectively) (Fig. 4b, c; closed arrowhead).

Taken together, these data suggest that loss of NETO1 selectively impairs the formation of KAR-containing synapses in GABAergic neurons while AMPAR synapses appear to differentiate normally. The loss of KAR-containing synapses in the Neto1KO cultures can fully explain the lower density of glutamatergic synapses, identified by PSD95-Syn co-localization in GAD67+ neurons (Fig. 4a).

\section{Loss of NET01 Has No Effect on AMPAR and NMDAR-Mediated Synaptic Inputs in CA3 Interneurons}

We went on to analyze whether absence of NETO1 affected functional glutamatergic input in CA3 stratum radiatum interneurons by recording spontaneous action potential independent miniature EPSC (mEPSC) at two different stages of development (P5 and P15). Both pharmacologically isolated AMPAR-KAR-mediated mEPSCs (mEPSC AMPA-KA $_{\text {And }}$ NMDAR-mediated mEPSC ( mEPSC $_{\text {NMDA }}$ ) were studied using hippocampal slices from WT and Neto1KO mice.

There was no significant difference between the genotypes in $\mathrm{mEPSC}_{\mathrm{AMPA}-\mathrm{KA}}$ at either developmental stage (frequency, P5 $0.33 \pm 0.07 \mathrm{~Hz}$ and $0.29 \pm 0.06 \mathrm{~Hz}$; P15 2.07 $\pm 0.22 \mathrm{~Hz}$ and $2.42 \pm 0.57 \mathrm{~Hz}$; amplitude P5 $26.3 \pm 2.7 \mathrm{pA}$ and $30.2 \pm 1.9$ $\mathrm{pA}$; P15 $20.5 \pm 1.0 \mathrm{pA}$ and $23.1 \pm 1.3 \mathrm{pA}$; for $\mathrm{WT}$ and Neto1KO, respectively) (Fig. 5a). Interestingly, application of the GluK1-selective agonist ACET (200 nM) significantly reduced $\mathrm{mEPSC}_{\mathrm{AMPA}-\mathrm{KA}}$ amplitude in P5 WT $(79.0 \% \pm 5.8 \%$ of control, $p=0.04$, Student's $t$ test) but not in Neto1KO interneurons $(93.7 \% \pm 4.3 \%$ of control) (Fig. 5b), supporting that immature CA3 interneurons contain NETO1-dependent synaptic KARs that have a minor contribution to postsynaptic current. In these experiments, no significant effect of ACET on the frequency of $\mathrm{mEPSC}_{\mathrm{AMPA}-\mathrm{KA}}$ was detected in either genotype $(119.7 \% \pm 15.1 \%$ and $87.3 \% \pm 9.9 \%$, for WT and Neto1KO, respectively).

Similar to mEPSC $_{\text {AMPA-KA, we detected no differences be- }}$ tween the genotypes in EPSC $_{\mathrm{NMDA}}$ at P5 (frequency $0.10 \pm$ $0.01 \mathrm{~Hz}$ and $0.11 \pm 0.02 \mathrm{~Hz}$, amplitude $19.4 \pm 0.8 \mathrm{pA}$ and $20.5 \pm 0.8 \mathrm{pA}$, for $\mathrm{WT}$ and Neto1KO, respectively). At P15, however, the mEPSC $_{\mathrm{NMDA}}$ amplitude was slightly higher in Neto1KO interneurons (WT $18.1 \pm 0.8$ pA, Neto1KO $20.8 \pm$ $1.0 \mathrm{pA}, p=0.04)$ while there was no difference between the genotypes in their frequency (WT $0.33 \pm 0.07 \mathrm{~Hz}$, Neto1KO $0.31 \pm 0.07 \mathrm{~Hz}$ ) (Fig. 5c).

NETO1 has been shown to be an interaction partner of NMDA receptors [7, 27] and to regulate the subunit composition of NMDAR at MF-CA3 synapses [7]. Therefore, we further tested the possibility that NETO1 affects NMDAR subunit composition in immature interneurons (P4-6). Application of ifenprodil $(5 \mu \mathrm{M})$, an antagonist selective for the NR2B-containing receptors, reduced the amplitude of evoked NMDAR-mediated EPSCs both in WT $(63.8 \% \pm$ $16.7 \%, n=5)$ and Neto1KO slices $(74.8 \% \pm 11.1 \%, n=9)$, but this effect was not significantly different between the genotypes (data not shown). Therefore, we concluded that NETO1 does not affect synaptic NMDARs in CA3 interneurons during early postnatal development.

Taken together, these data show that loss of NETO1 has no major effects on AMPAR- and NMDAR-mediated synaptic transmission in CA3 interneurons.

\section{Loss of NET01 Has Minor or No Effects on the Excitability of Immature Hippocampal Network}

Hippocampal KARs have been shown to modulate early network activity [19, 32, 33], characterized by spontaneously occurring network bursts [34] that are highly dependent on intact excitation-inhibition balance [35]. To evaluate the significance of NETO1 in regulation of network excitability in the immature hippocampus, we first recorded spontaneous action potential (AP) firing of CA3 stratum radiatum interneurons using cell-attached recordings from P3 and P10 WT and Neto1KO mice (Fig. 6a). At P3-6, we observed a large heterogeneity in the firing frequency of individual recorded cells in both WT and Neto1KO, but no significant differences between genotypes (mean AP frequency, WT $2.82 \pm 0.46 \mathrm{~Hz}$; Neto1KO $2.42 \pm 0.38 \mathrm{~Hz}$ ) (Fig. 6b, c). The interneuron subtypes become more differentiated by P10 with some cells 
a
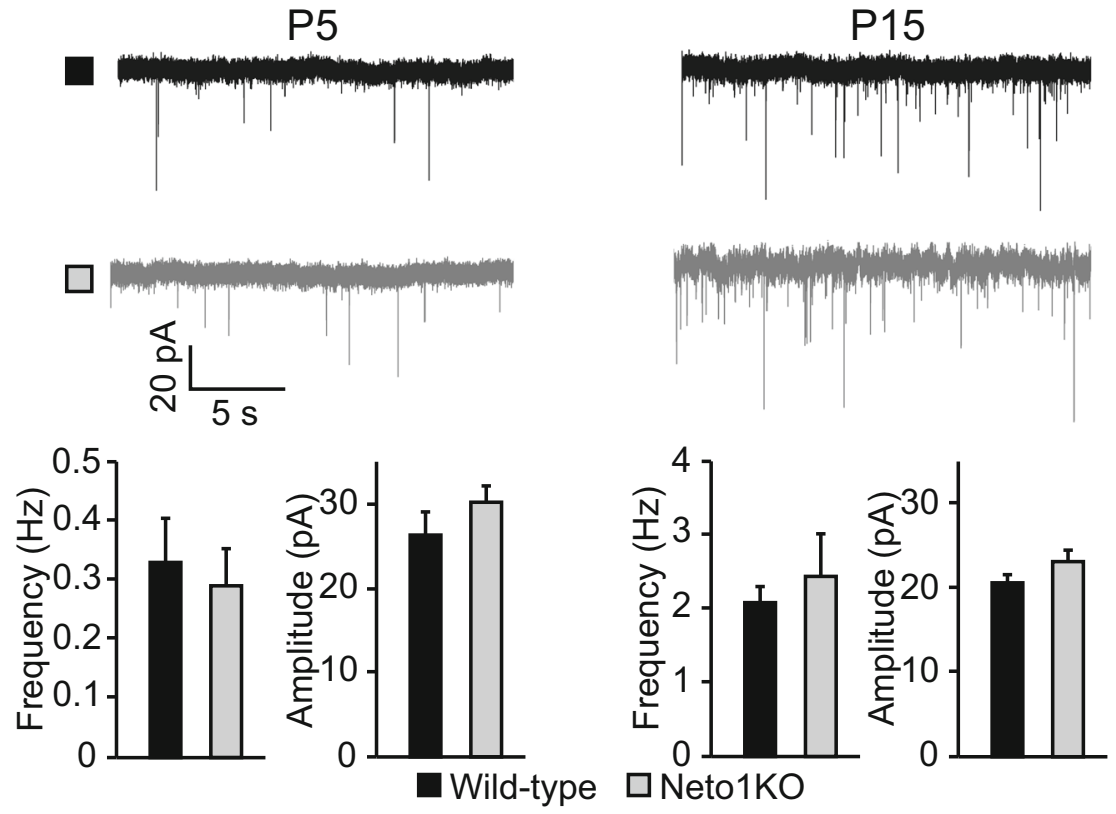

b
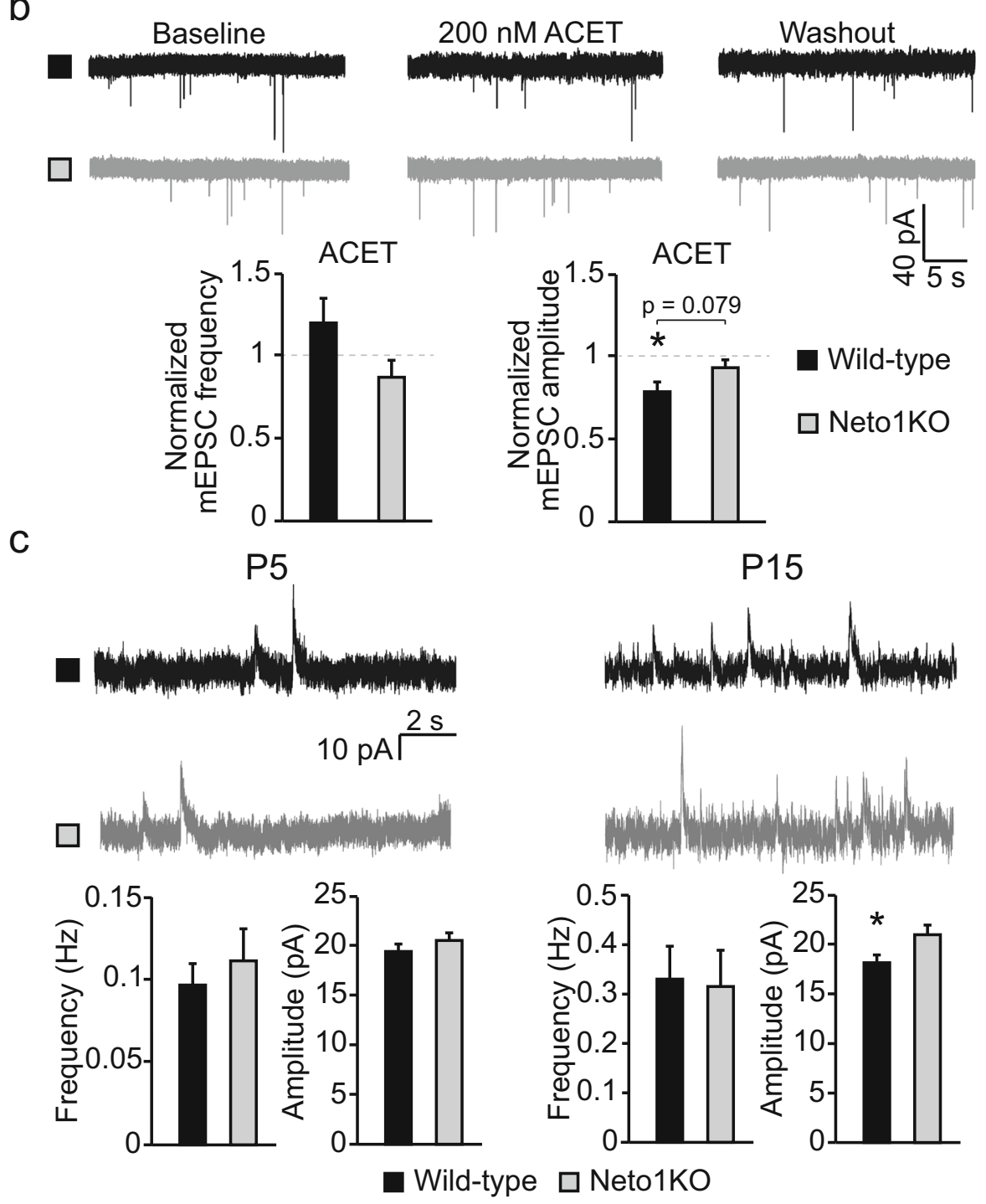
Fig. 5 NETO1 deficiency has no significant effect on the development of AMPAR- or NMDAR-mediated synaptic input into CA3 stratum radiatum interneurons. a Example traces and pooled data illustrating AMPAR-mediated $\mathrm{mEPSCs}$ recorded from $\mathrm{CA} 3$ stratum radiatum interneurons in WT (black, $n=12, n=17$ ) and Neto1 KO (gray, $n=14, n=15$ ) slices from P4-6 to P14-16 mice, respectively. b Example traces and quantified data illustrating the effect of GluK1-selective antagonist ACET $(200 \mathrm{nM})$ on mEPSC frequency and amplitude in WT $(n=8)$ and Neto1KO $(n=6)$ P4-6 CA3 stratum radiatum interneurons. c Example traces and pooled data depicting NMDAR-mediated mEPSCs recorded from CA3 stratum radiatum interneurons in WT (black, $n=13$, $n=14$ ) and Neto1KO (gray, $n=9, n=13$ ) slices from P4-6 and P14-16 mice, respectively. $* p<0.05 ; * * p<0.01 ; * * * p<0.001$

acquiring distinctive high action potential firing frequency. The mean AP firing frequency was not different between the genotypes at P10 when all cells were included in the analysis $(3.27 \pm 0.78 \mathrm{~Hz}, n=12 ; 2.56 \pm 0.69 \mathrm{~Hz}, n=14$; WT and Neto1KO, respectively). However, if the cells with high (> $5 \mathrm{~Hz}$ ) firing frequency were excluded from the analysis, we observed a significantly slower spontaneous firing of Neto1KO interneurons $(1.51 \pm 0.28 \mathrm{~Hz}, p=0.02)$ as compared to WT $(2.43 \pm 0.19 \mathrm{~Hz})$ at P10 (Fig. 6b, c).

To further study the role of NETO1 in neonatal network activity, we recorded spontaneous synaptic currents from WT and Neto1KO CA3 pyramidal cells that receive GABAergic inputs from $\mathrm{CA} 3$ interneurons. By using a low chloride concentration in the pipette filling solution, we were able to analyze the frequency of network bursts, spontaneous inhibitory (sIPSC) and spontaneous excitatory postsynaptic currents (sEPSC) from the same recordings. The basal frequency of network bursts was not different between the genotypes at P4-6 (WT $0.032 \pm 0.003 \mathrm{~Hz}$; Neto1KO $0.029 \pm 0.002 \mathrm{~Hz}$ ) (Fig. 7a, b). Consistent with the data showing no difference in the interneuron firing frequency, the frequency of sIPSCs in the CA3 pyramidal neurons was similar in WT and Neto1KO slices $(1.46 \pm 0.84 \mathrm{~Hz}$ and $1.28 \pm 0.16 \mathrm{~Hz}$, respectively). However, sIPSC amplitude was lower in Neto1KO (17.1 \pm $0.6 \mathrm{pA})$ as compared to WT $(21.2 \pm 1.0 \mathrm{pA}, p=0.005)$ (Fig. $7 b$ ). Lastly, sEPSC frequency, but not amplitude, was significantly lower in Neto1KO CA3 pyramidal cells as compared to WT (frequency $0.23 \pm 0.03 \mathrm{~Hz}$ and $0.13 \pm 0.01 \mathrm{~Hz}, p=$ 0.04 ; amplitude $22.3 \pm 1.7 \mathrm{pA}$ and $19.7 \pm 1.2 \mathrm{pA}$, for WT and Neto1KO, respectively) (Fig. 7b).

Thus, NETO1 deficiency had minor or no effects on the spontaneous network activity in area CA3 during the first week of life. Despite its strong effects on interneuronal KARs, NETO1 is not indispensable for physiological network activity, and the immature circuit lacking NETO1 is able to maintain the excitability similar to that in the WT.

\section{NET01-Deficient Network Is Less Sensitive to Kainate}

Since the excitability of the neonatal hippocampal network is under strong homeostatic control [36], mechanisms that a

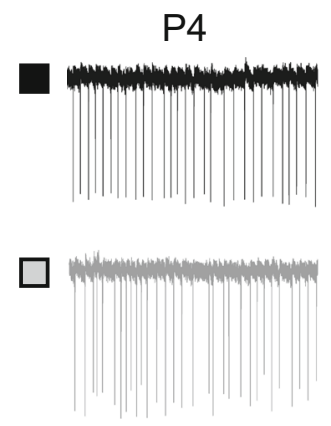

b
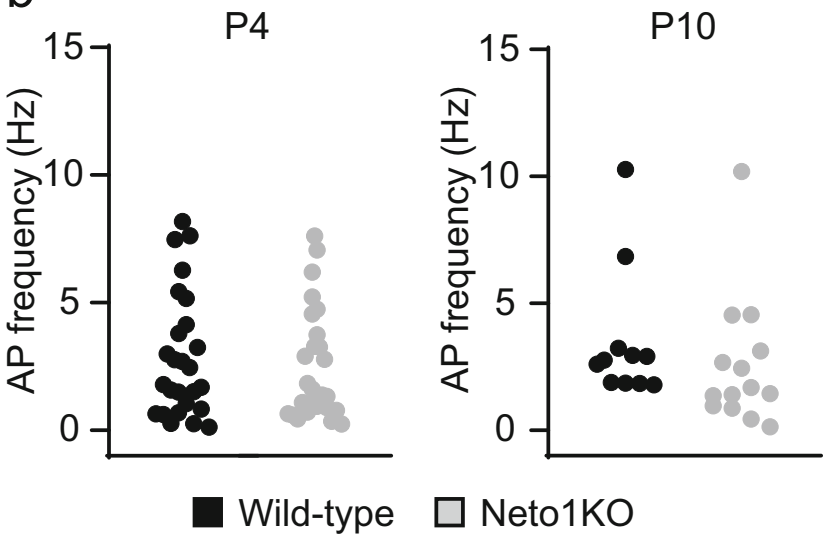

C

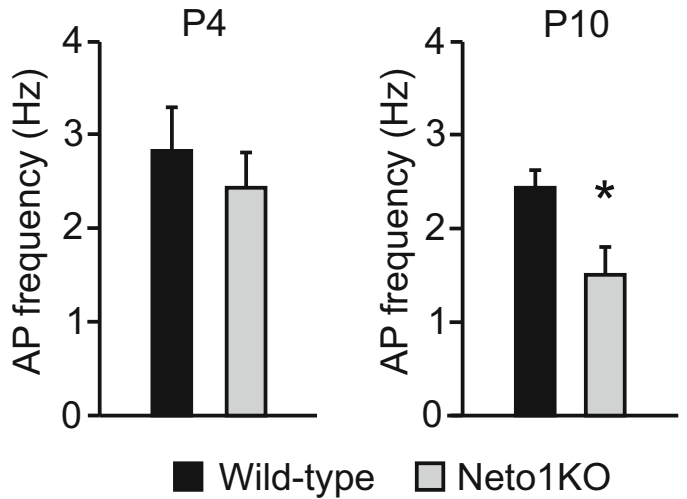

Fig. 6 Spontaneous action potential firing of CA3 stratum radiatum interneurons in WT and Neto1KO slices. a Example traces of cellattached recordings illustrating spontaneous action potential firing of CA3 stratum radiatum interneurons of WT (black) and Neto1KO (gray) slices at P4 and P10. b Graphs showing the mean action potential firing frequency of individual CA3 stratum radiatum interneurons at P3-6 $(n=18, n=17)$ and P9-10 $(n=12, n=14)$ in WT and Neto1KO slices, respectively. c Pooled data of mean action potential firing frequency of CA3 stratum radiatum interneurons at $\mathrm{P} 3-6(n=18, n=17)$ and $\mathrm{P} 9-10(n=9, n=11)$ from WT and Neto1KO, respectively. Cells with high firing frequency are excluded from the P9-10 age group. * $p<0.05 ; * * p<0.01 ; * * * p<0.001$

modulate circuit activity might be compensated for in a knockout model and thus not observed under basal conditions. Therefore, we went on to investigate whether the NETO1deficient network was able to respond to low concentration 

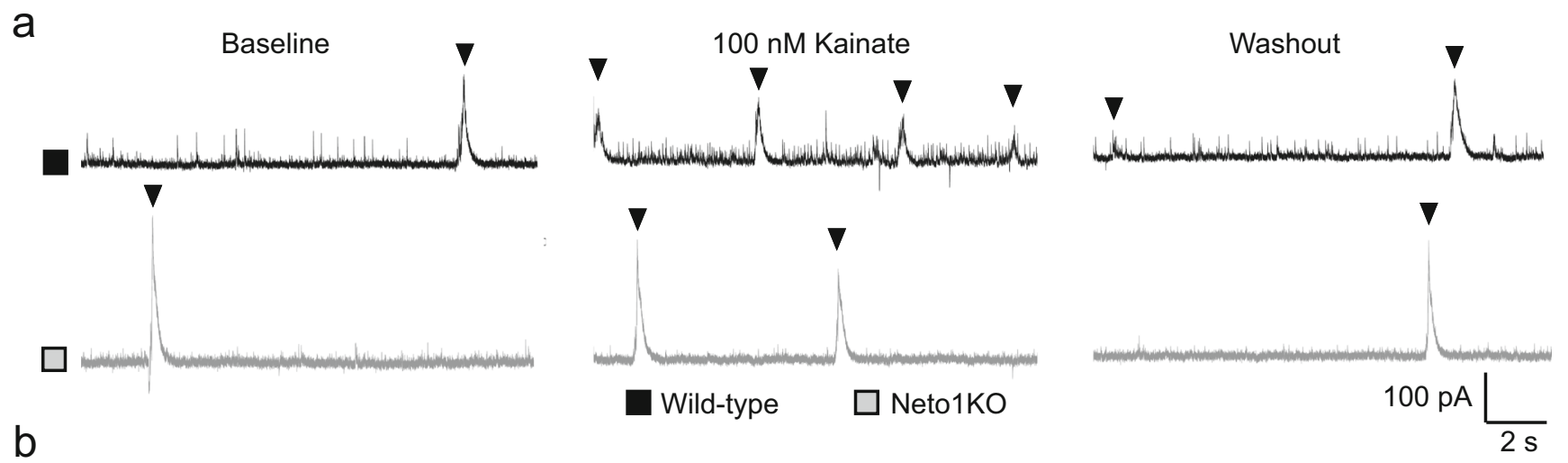

b

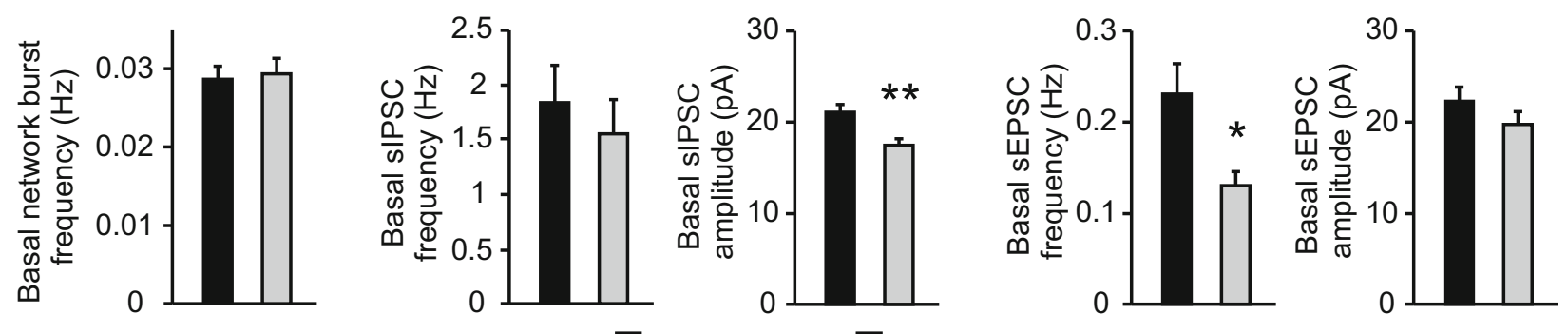

C

Wild-type

$\square$ Neto1KO
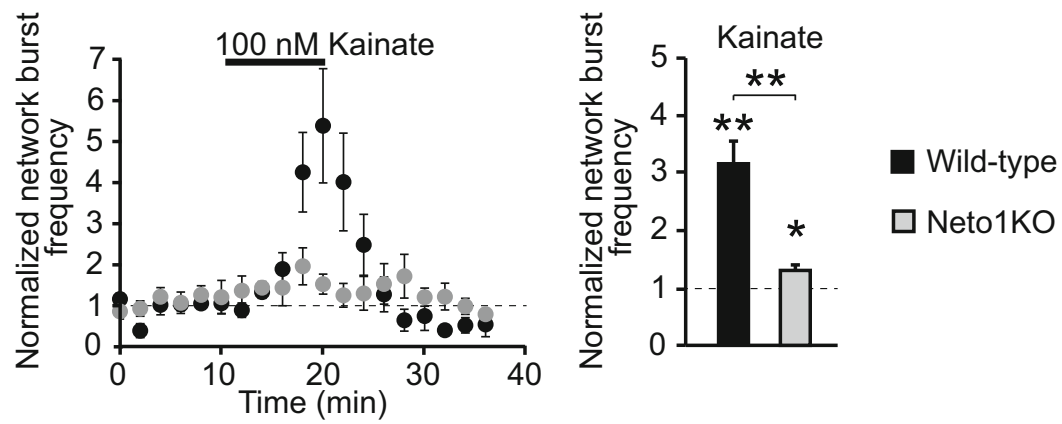

d
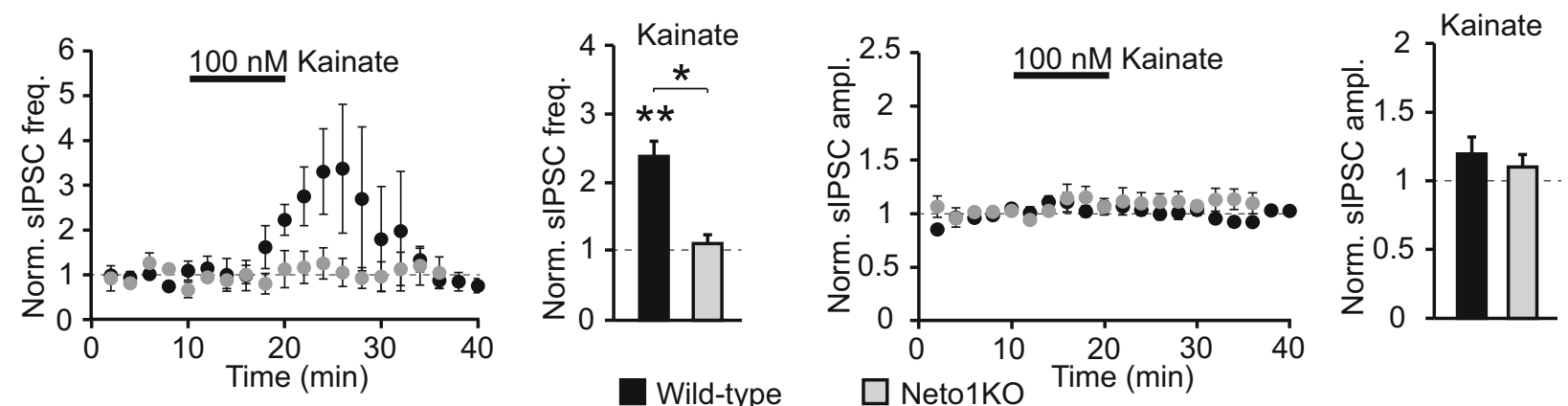

e
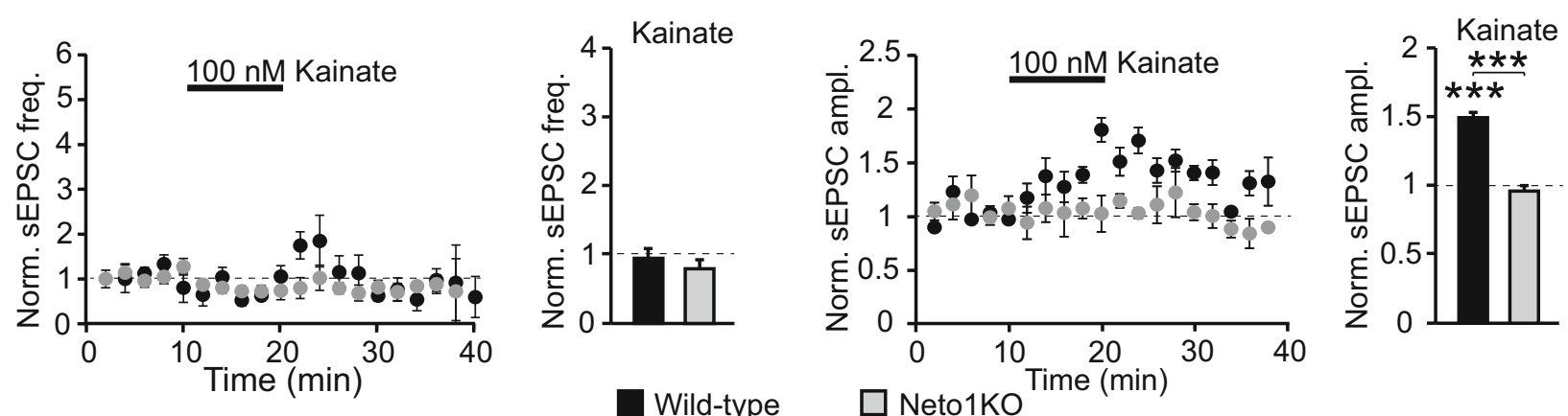
Fig. 7 NETO1/KAR complex increases the kainate sensitivity of the immature CA3 network. a Example traces illustrating the effect of $100 \mathrm{nM}$ kainate (KA) on spontaneous network activity recorded from P5 CA3 pyramidal neurons in WT and Neto1KO slices. With the use of low chloride electrode filling solution, GABAergic and glutamatergic synaptic events are seen as outward and inward currents, respectively. The network bursts are indicated with black arrowheads. b Pooled data illustrating the frequency of network bursts $(n=29, n=30), \operatorname{sIPSCs}(n=$ $14, n=14)$, and sEPSCs $(n=14, n=14)$ as well as the amplitude of sIPSCs and sEPSC in P4-6 CA3 principal neurons in WT (black) and Neto1KO (gray) slices, respectively. c Time course plot and pooled data demonstrating the effect of $100 \mathrm{nM}$ kainate on network bursts in CA3 pyramidal neurons of P4-6 WT $(n=7)$ and Neto1KO $(n=5)$ mice. d Time course plots and pooled data showing effect of $100 \mathrm{nM}$ kainate on sIPSC frequency and amplitude in CA3 pyramidal neurons of P4-6 WT $(n=6)$ and Neto1KO $(n=3)$ mice. e Corresponding data for sEPSCs (WT, $n=7$; Neto1KO, $n=4)$. * $p<0.05 ; * * p<0.01 ; * * * p<0.001$

of kainate (KA), which potently increases excitability of the hippocampal network already during the first postnatal week [33].

As expected, application of $100 \mathrm{nM} \mathrm{KA}$ resulted in a robust increase in the occurrence of network bursts in CA3 pyramidal neurons in WT slices (P4-6) $(313.6 \% \pm 39.7 \%$ of baseline, $p=0.001$, Student's $t$ test) (Fig. 7a, c). In the Neto1KO slices, $100 \mathrm{nM}$ KA induced a small increase in the frequency of network bursts $(129.3 \% \pm 9.0 \%$ of baseline, $p=0.02$, Student's $t$ test) (Fig. 7a, c). However, KA effect on the burst frequency in WT was significantly larger as compared to Neto1KO ( $p=0.003$, Kruskal-Wallis) (Fig. 7a, c).

In addition to the increase in the network bursts, $100 \mathrm{nM}$ KA caused a large increase in the frequency of sIPSCs $(237.6 \% \pm 41.7 \%$ of baseline, $p=0.007$, Student's $t$ test) in WT slices but had no significant effect on sIPSC amplitude $(119.6 \% \pm 9.1 \%$ of baseline) (Fig. $7 d)$. Consistent with the loss of somatodendritic ionotropic KARs in interneurons, $\mathrm{KA}$ had no effect on sIPSCs in Neto1KO slices (frequency $110.9 \% \pm 37.2 \%$ of baseline; amplitude $110.2 \% \pm 8.9 \%$ of baseline) (Fig. 7d). Analysis of spontaneous glutamatergic currents indicated an increase in sEPSC amplitude in response to KA application in WT $(150.1 \% \pm 6.0 \%$ of baseline, $p<0.001$, Student's $t$ test) but not in Neto $1 \mathrm{KO}$ slices (94.7\% $\pm 5.3 \%$ of baseline; $p<0.001$ between genotypes) (Fig. 7e). KA had no effect on sEPSC frequency either in WT $(94.0 \% \pm 14.0 \%$ of baseline $)$ or Neto1KO $(79.8 \% \pm$ $12.9 \%$ of baseline) (Fig. 7e).

These data provide evidence that NETO1 is critical for KAinduced network bursts and participates in recruiting the inhibitory drive from immature GABAergic interneurons to CA3 pyramidal cells.

\section{Discussion}

KARs have developmentally restricted functions in the hippocampus both in principal cells $[21,26,31]$ and in interneurons
$[19,37]$. We have previously shown that NETO1 is critical for the immature-type KAR functions and maturation of the connectivity between CA3-CA1 principal neurons [21], while the role of NETOs at GABAergic interneurons in the neonatal hippocampus is not previously characterized. In the present study, we provide the first description of NETO1-dependent subcellular targeting of KAR subunits in GABAergic interneurons. Furthermore, we show that NETO1 regulates both ionotropic and metabotropic KAR functions in CA3 interneurons already during the first week of life and is critical for KAinduced modulation of network bursts and GABAergic transmission at the immature network.

\section{NETO1 and Subcellular Targeting of KARs at GABAergic Neurons}

NETO1 is expressed in interneurons in the neonatal [21] and adult hippocampus $[5,22]$, where it is co-expressed with KAR subunits GluK1, 2, and 5 in different interneuron subpopulations [22]. While the role of NETO1 in targeting of various KAR subunits has been studied in glutamatergic neurons [5-7, 21, 38-40], no previous data from GABAergic neurons exists.

Our data show that a predominant effect of NETO1 on interneuronal KARs is to promote their dendritic and axonal targeting, which was significantly impaired in NETO1deficient GABAergic neurons irrespective of the KAR subunit identity. We recently reported a similar role for NETO1 in axons of hippocampal principal neurons [21]. In contrast to NETO1, NETO2 appears to not be involved in axonal and dendritic delivery of interneuronal KAR subunits, at least under our culture conditions.

In the principal neurons, NETO1 is also reported to promote the postsynaptic capture of GluK1 and GluK2 [6, 7] or selectively GluK1 [39, 40]. Here, we observed no difference in synaptic recruitment of KAR subunits GluK1b and GluK2 between WT and NETO1-deficient interneurons. In contrast, the synaptic distribution of GluK1c subunit, which is endogenously mainly expressed in principal neurons of the immature hippocampus, was significantly impaired in the Neto1KO interneurons. These data suggest that NETO1 has subunit and cell-type specific effects on KAR trafficking at the level of synapses [38, 40], while promoting distal targeting of KARs in a subunit independent manner in both glutamatergic and GABAergic neurons.

\section{NET01/KAR-Dependent Synaptic Signaling in Immature Interneurons}

Functional characterization of immature CA3 interneurons indicated that both ionotropic and metabotropic KAR signaling were compromised or completely lost in the absence of NETO1, similar to that previously shown for CA3 principal 
neurons in adult hippocampus [5-7]. Thus, we identified a small KAR-mediated component of EPSC in the WT but not Neto1KO interneurons in response to $50 \mathrm{~Hz} / 5$ pulse afferent stimulation. Also, the G protein coupled regulation of $I_{\mathrm{mAHP}}$ via GluK1 subunit containing KARs was not observed in NETO1-deficient interneurons. Intriguingly, however, agonist application revealed a subpopulation of functional ionotropic GluK1-containing KARs in NETO1-deficient CA3 stratum radiatum interneurons supporting the idea that NETO1 has distinct effects on different types of KARs even within one neuron.

Postsynaptic KAR-mediated current has been previously described in adult CA3 pyramidal cells $[41,42]$ and in CA1 interneurons $[10,17]$. In addition, there is evidence suggesting that KARs and AMPARs in CA1 interneurons are located at distinct synapse populations [15]. Interestingly, in CA1 pyramidal cells, NETO1 overexpression targets KARs preferentially to synapses that contain no AMPARs [39], providing a plausible mechanism for generation of KAR-containing AMPAR-lacking synapses in GABAergic interneurons where NETO1 is endogenously expressed. Indeed, analysis of AMPAR- and KAR-containing synaptic clusters in cultured GABAergic neurons identified synapses that contained only AMPAR, only KAR, but also synapses with both AMPARs and KARs. In the absence of NETO1, the density of both KAR-only and AMPAR-KAR synapses were reduced, while AMPAR-containing synapses were not affected. It should be noted that these data were obtained using overexpression of recombinant KAR subunits in cell culture, which might override some endogenous targeting mechanisms. However, also functional analysis supported existence of NETO1-dependent postsynaptic KARs in immature CA3 interneurons. Therefore, the most likely interpretation of these results is that ionotropic NETO1/KAR signaling operates in a small fraction of synapses in the CA3 interneurons during early postnatal development and exhibits a rather modest contribution to postsynaptic current.

\section{NET01 Regulates Formation of KAR-Containing Synapses but Has No Effect on Maturation of AMPAR- and NMDAR-Mediated Transmission in Interneurons}

KARs have been implicated in synaptogenesis and synaptic maturation in the CA1 circuitry $[21,24,26,31]$ as well as in the mossy fiber-CA3 synapse $[23,25]$. In addition, KARs regulate maturation of the dendritic tree in both, principal neurons and interneurons [43, 44]. Our data from cell cultures showed that loss of NETO1 is associated with compromised synaptogenesis that can be rescued with overexpression of KARs in GABAergic neurons. However, this effect could be fully explained by the loss of KAR-containing synapses, while AMPAR-containing synapses formed apparently normally in the absence of NETO1.
The rescue of KAR-synapses in NETO1-deficient GABAergic neurons was subunit dependent. GluK1c overexpression was not able to rescue the impaired synapse formation in the Neto1KO, most likely because it is not efficiently recruited to postsynaptic compartments in GABAergic Neto1KO neurons. However, overexpression of GluK1b and GluK2 subunits rescued the overall density of synapses in the Neto1KO GABAergic neurons to the WT level. Since NETO1 had no effect on the synaptic distribution of GluK1b and GluK2 in the GABAergic neurons, the loss of KARcontaining synapses in the NetolKO is likely due to the impaired dendritic delivery of KAR subunits which limits the number of available receptors at the synaptic site. In support to this idea, overexpression of GluK2 subunit in the WT was associated with a significantly higher density of KARcontaining synapses as compared to GluK1b, which is not delivered to dendrites as efficiently as GluK2.

Consistent with the lack of effect of NETO1 on synaptic AMPAR clusters in cultured interneurons, we found no significant differences in the AMPAR-mediated transmission to WT vs Neto1KO interneurons in the area CA3 of neonatal mice. Interestingly, NMDAR transmission exhibited NETO1-dependent phenotype by the end of the second postnatal week. Therefore, we tested for the possibility that NETO1 regulates the NMDAR subunit composition in the interneurons, similar to that shown for CA3 principal cells [7], which could consequently affect development of glutamatergic inputs to interneurons [45]. However, no difference in the ifenprodil sensitivity of NMDAR-mediated current was detected between the genotypes.

Thus, while our data identify a role for NETO1 in promoting formation of KAR-containing synapses, lack of NETO1/ KAR signaling had no apparent consequences on development and maturation of AMPAR-NMDAR-mediated synaptic transmission in GABAergic interneurons. Together with previous data $[21,23-26,31]$, these results support that KARs regulate development of glutamatergic transmission in a cell type specific manner, promoting maturation of AMPAR-mediated transmission in principal glutamatergic neurons, but not in GABAergic interneurons. Further, our data suggest that KAR-synapses at GABAergic interneurons are not developmental precursors of AMPA-containing synapses but rather represent a distinct population of synapses, whose physiological significance at the immature circuitry remains elusive.

\section{NET01/KAR Complex Has Little or No Effect on the Excitability of the Immature Hippocampal Network but Is Required for KA Modulation}

KARs are potent regulators of interneuron excitability in the neonatal hippocampus [19, 32, 37]. However, we observed no effect of NETO1 on the basal spontaneous action potential 
firing of CA3 interneurons during the first week of postnatal development, in contrast to significantly lower firing of GluK1KO interneurons at the same developmental stage [19]. Consistently, under basal conditions, NETO1 deficiency had no effect on the medium after hyperpolarizing current, proposed to underlie the differences AP firing frequency in neonatal GluK1-lacking interneurons [19]. The excitability of the neonatal network is under strict homeostatic control [36, 46-48]; thus, it is feasible that any NETO1-dependent mechanisms regulating action potential firing frequency are compensated for and thus not manifested in a knockout model.

Interestingly, during the second postnatal week and in parallel with maturation of interneuronal firing properties, the spontaneous AP firing frequency became lower in NETO1deficient CA3 interneurons as compared to WT controls. Possibly, at the mature network, postsynaptic NETO1/KAR signaling controls temporal summation of EPSCs and thereby contributes to spike generation in $\mathrm{CA} 3$ interneurons, similar to that shown for CA3 pyramidal neurons $[5,49]$ and CA1 interneurons [18]. Alternatively, the altered firing frequency in the Neto1KO interneurons could reflect some developmental delay in their maturation [44].

In accordance with the unchanged interneuron firing during the first postnatal week, immature-type spontaneous network activity, recorded from CA3 pyramidal neurons, was not markedly affected by the absence of NETO1. The main observed effect, i.e., a significant reduction in the frequency of sEPSCs, is likely due to loss of presynaptic KARs that tonically facilitate glutamate release at this developmental stage $[21,31,32]$. However, similar to that shown previously in adult Neto1KO [22], the immature NETO1-deficient CA3 network was strikingly less sensitive to KA modulation. Application of $100 \mathrm{nM}$ KA had only modest effects in the Neto1KO in contrast to the robust induction of network bursts in the WT [33]. KA activates KARs in various subcellular compartments in the pyramidal neurons and interneurons, and both cell types express NETO1 in the neonate CA3. Our data do not resolve which KAR population is mainly responsible for the loss of KA-dependent network bursts in the Neto1KO. However, previous data have identified that low concentration of KA induce ectopic spiking of CA3 pyramidal neurons that initiates network bursts in the neonatal hippocampus [33], suggesting that loss of axonal KARs in Neto1KO CA3 pyramidal neurons [21] contributes to this phenotype.

KA application in the CA3 network also associates with a large increase in the frequency of spontaneous GABAergic events, an effect that has been attributed to KAR-mediated depolarization of GABAergic neurons and their axons as well as regulation of GABA release $[1-3,8,9]$. This effect is strongly impaired in adult NETO1-deficient mice [22], indicating a central role for NETO1 in recruitment of GABAergic inputs to CA3 pyramidal neurons in response to KA application.
Consistent with these previous findings, we found that the effect of $100 \mathrm{nM}$ KA on sIPSCs in the immature CA3 network was lost in Neto1KOs. Interestingly, axonal delivery of KAR subunits was significantly impaired in cultured Neto1KO GABAergic neurons, suggesting that loss of axonal and presynaptic KARs, together with the deficit in somatodendritic KARs, contributes to the low KA sensitivity of GABAergic transmission in the Neto1KO. In support to this idea, it was recently reported that NETO1 is required for presynaptic KAR function at CCK/CB1 interneurons in the adult hippocampus [22]. However, we cannot rule out the possibility that reduced affinity of KARs in the absence of NETO1 also contributes to the observed phenotype $[5,50,51]$.

Taken together, these findings support that while NETO1 is required for many of the functions ascribed to KARs in immature CA3 interneurons, NETO1 deficiency does not have severe consequences on the basal excitability of the CA3 network during early postnatal development. However, NETO1 is central for KA-dependent modulation of the network activity and GABAergic synaptic transmission already during the first week of life. Given that aberrant KAR-mediated transmission has been implicated in certain forms of epilepsy [52], NETO1 might provide an attractive target for development of novel treatments against adult and early life seizures.

Acknowledgments We thank Roderick McInnes and David Ng (McGill University, Montreal, Canada) for providing the Neto1 and Neto2 knockout mouse lines. We acknowledge Kirsi Kolehmainen for excellent technical help. Special thanks to the Light Microscopy Unit in University of Helsinki for their expert advice and technical assistance in confocal imaging and analysis.

Funding Information Open access funding provided by University of Helsinki. This work was supported by the Academy of Finland, Jane and Aatos Erkko Foundation, and Sigrid Juselius Foundation.

Compliance with Ethical Standards The animal experiments were performed in accordance with the University of Helsinki Animal Welfare Guidelines.

Conflict of Interest The authors declare that they have no conflict of interest.

Open Access This article is distributed under the terms of the Creative Commons Attribution 4.0 International License (http:// creativecommons.org/licenses/by/4.0/), which permits unrestricted use, distribution, and reproduction in any medium, provided you give appropriate credit to the original author(s) and the source, provide a link to the Creative Commons license, and indicate if changes were made.

\section{References}

1. Lerma J, Marques JM (2013) Kainate receptors in health and disease. Neuron 80(2):292-311 
2. Carta M, Fièvre S, Gorlewicz A, Mulle C (2014) Kainate receptors in the hippocampus. Eur J Neurosci 39:1835-1844

3. Evans AJ, Gurung S, Henley JM, Nakamura Y, Wilkinson KA (2017) Exciting times: new advances toward understanding the regulation of roles of kainate receptors. Neurochem Res 44:572584. https://doi.org/10.1007/s11064-017-2450-2

4. Copits BA, Swanson GT (2012) Dancing partners at the synapse: auxiliary subunits that shape kainate receptor function. Nat Rev Neurosci 13(10):675-686

5. Straub C, Hunt DL, Yamasaki M, Kim KS, Watanabe M, Castillo PE, Tomita S (2011) Distinct functions of kainate receptors in the brain are determined by the auxiliary subunit Neto1. Nat Neurosci 14(7):866-873

6. Tang M, Pelkey KA, Ng D, Ivakine E, McBain CJ, Salter MW, McInnes RR (2011) Neto1 is an auxiliary subunit of native synaptic kainate receptors. J Neurosci 31(27):10009-10018

7. Wyeth MS, Pelkey KA, Petralia RS, Salter MW, McInnes RR, McBain CJ (2014) Neto auxiliary protein interactions regulate kainate and NMDA receptor subunit localization at mossy fiberCA3 pyramidal cell synapses. J Neurosci 34(2):622-628

8. Kullmann DM (2001) Presynaptic kainate receptors in the hippocampus: slowly emerging from obscurity. Neuron 32(4):561-564

9. Akgül G, McBain CJ (2016) Diverse roles for ionotropic glutamate receptors on inhibitory interneurons in developing and adult brain. $\mathrm{J}$ Physiol 594(19):5471-5490

10. Cossart R, Esclapez M, Hirsch JC, Bernard C, Ben-Ari Y (1998) GluR5 kainate receptor activation in interneurons increases tonic inhibition of pyramidal cells. Nat Neurosci 1(6):470-478

11. Paternain A, Herrera M, Angela Nieto M, Lerma J (2000) GluR5 and GluR6 kainate receptor subunits coexist in hippocampal neurons and coassemble to form functional receptors. J Neurosci 20(1): 196-205

12. Mulle C, Sailer A, Swanson GT, Brana C, O'Gorman S, Bettler B, Heinemann SF (2000) Subunit composition of kainate receptors in hippocampal interneurons. Neuron 28:475-484

13. Fisahn A, Contractor A, Traub RD, Buhl EH, Heinemann SF, McBain C (2004) Distinct roles for the kainate receptor subunits GluR5 and GluR6 in kainate-induced hippocampal gamma oscillations. J Neurosci 24(43):9658-9668

14. Christensen JK, Paternain AV, Selak S, Ahring PK, Lerma J (2004) A mosaic of functional kainate receptors in hippocampal interneurons. J Neurosci 24(41):8986-8993

15. Wondolowski J, Frerking M (2009) Subunit-dependent postsynaptic expression of kainate receptors on hippocampal interneurons in area CA1. J Neurosci 29(2):563-574

16. Vesikansa A, Sakha P, Kuja-Panula J, Molchanova S, Rivera C, Huttunen HJ, Rauvala H, Taira T et al (2012) Expression of GluK1c underlies the developmental switch in presynaptic kainate receptor function. Sci Rep 2:310

17. Frerking M, Malenka RC, Nicoll RA (1998) Synaptic activation of kainate receptors on hippocampal interneurons. Nat Neurosci 1(6): 479-486

18. Frerking M, Ohliger-Frerking P (2002) AMPA receptors and kainate receptors encode different features of afferent activity. $\mathrm{J}$ Neurosci 22:7434-7443

19. Segerstråle M, Juuri J, Lanore F, Piepponen P, Lauri SE, Mulle C, Taira T (2010) High firing rate of neonatal hippocampal interneurons is caused by attenuation of after hyperpolarizing potassium currents by tonically active kainate receptors. J Neurosci 30(19): $6507-6514$

20. Tomita S, Castillo PE (2012) Neto1 and Neto2: auxiliary subunits that determine key properties of native kainate receptors. J Physiol 590(10):2217-2223

21. Orav E, Atanasova T, Shintyapina A, Kesaf S, Kokko M, Partanen J, Taira T, Lauri SE (2017) NETO1 dependent axonal targeting of kainate receptors is critical for development of glutamatergic connectivity in the hippocampus. eNeuro 4(3)

22. Wyeth MS, Pelkey KA, Yuan X, Vargish G, Johnston AD, Hunt S, Fang C, Abebe D et al (2017) Neto auxiliary subunits regulate interneuron somatodendritic and presynaptic kainate receptors to control network inhibition. Cell Rep 20(9):2156-2168

23. Marchal C, Mulle C (2004) Postnatal maturation of mossy fibre excitatory transmission in mouse CA3 pyramidal cells: a potential role for kainate receptors. J Physiol 561:27-37

24. Vesikansa A, Sallert M, Taira T, Lauri SE (2007) Long-term activation of kainate receptor subunit GluR5 increases mEPSC frequency in the hippocampal slice cultures. J Physiol 583:145-157

25. Lanore F, Labrousse VF, Szabo Z, Normand E, Blanchet C, Mulle C (2012) Deficits in morphofunctional maturation of hippocampal mossy fiber synapses in a mouse model of intellectual disability. $\mathrm{J}$ Neurosci 32:17882-17893. https://doi.org/10.1523/JNEUROSCI. 2049-12.2012

26. Sakha P, Vesikansa A, Orav E, Heikkinen J, Kukko-Lukjanov TK, Shintyapina A, Franssila S, Jokinen V et al (2016) Axonal kainate receptors modulate the strength of efferent connectivity by regulating presynaptic differentiation. Front Cell Neurosci 10:3

27. Ng D, Pitcher GM, Szilard RK, Sertié A, Kanisek M, Clapcote SJ, Lipina T, Kalia LV et al (2009) Neto1 is a novel CUB-domain NMDA receptor-interacting protein required for synaptic plasticity and learning. PLoS Biol 7(2):e41

28. Schmitz SK, Hjorth JJ, Joemai RM, Wijntjes R, Eijgenraam S, de Bruijn P, Georgiou C, de Jong AP et al (2011) Automated analysis of neuronal morphology, synapse number and synaptic recruitment. J Neurosci Methods 195(2):185-193

29. Anderson WW, Collingridge GL (2007) Capabilities of the WinLTP data acquisition program extending beyond basic LTP experimental functions. J Neurosci Methods 162(1-2):346-356

30. Clarke VR, Collingridge GL, Lauri SE, Taira T (2012) Synaptic kainate receptors in CA1 interneurons gate the threshold of thetafrequency-induced long-term potentiation. J Neurosci 32(50): 18215-18226

31. Lauri SE, Vesikansa A, Segerstråle M, Collingridge GL, Isaac JT, Taira T (2006) Functional maturation of CA1 synapses involves activity-dependent loss of tonic kainate receptor-mediated inhibition of glutamate release. Neuron 50:415-429

32. Lauri SE, Segerstråle M, Vesikansa A, Maingret F, Mulle C, Collingridge GL, Isaac JT, Taira T (2005) Endogenous activation of kainate receptors regulates glutamate release and network activity in the developing hippocampus. J Neurosci 25(18):4473-4484

33. Juuri J, Clarke VRJ, Lauri SE, Taira T (2010) Kainate receptorinduced ectopic spiking of CA3 pyramidal neurons initiates network bursts in neonatal hippocampus. J Neurophys 104:1696-1706

34. Ben-Ari Y (2001) Developing networks play a similar melody. Trends Neurosci 24(6):353-360

35. Lamsa K, Palva JM, Ruusuvuori E, Kaila K, Taira T (2000) Synaptic GABA(A) activation inhibits AMPA-kainate receptormediated bursting in the newborn (P0-P2) rat hippocampus. $\mathrm{J}$ Neurophysiol 83(1):359-366

36. Huupponen J, Molchanova SM, Taira T, Lauri SE (2007) Susceptibility for homeostatic plasticity is down-regulated in parallel with maturation of the rat hippocampal synaptic circuitry. $\mathrm{J}$ Physiol 581(Pt 2):505-514

37. Maingret F, Lauri SE, Taira T, Isaac JT (2005) Profound regulation of neonatal CA1 rat hippocampal GABAergic transmission by functionally distinct kainate receptor populations. J Physiol 567(Pt 1):131-142

38. Copits BA, Robbins JS, Frausto S, Swanson GT (2011) Synaptic targeting and functional modulation of GluK1 kainate receptors by the auxiliary neuropilin and tolloid-like (NETO) proteins. J Neurosci 31(20):7334-7340 
39. Sheng N, Shi YS, Lomash RM, Roche KW, Nicoll RA (2015) Neto auxiliary proteins control both the trafficking and biophysical properties of the kainate receptor GluK1. Elife 4:e11682

40. Sheng N, Shi YS, Nicoll RA (2017) Amino-terminal domains of kainate receptors determine the differential dependence on Neto auxiliary subunits for trafficking. Proc Natl Acad Sci U S A 114(5):1159-1164

41. Castillo PE, Malenka RC, Nicoll RA (1997) Kainate receptors mediate a slow postsynaptic current in hippocampal CA3 interneurons. Nature 388(6638):182-186

42. Vignes M, Collingridge GL (1997) The synaptic activation of kainate receptors. Nature 388:179-182

43. Marques JM, Rodrigues RJ, Valbuena S, Rozas JL, Selak S, Marin P, Aller MI, Lerma J (2013) CRMP2 tethers kainate receptor activity to cytoskeleton dynamics during neuronal maturation. $\mathrm{J}$ Neurosci 33:18298-18310

44. Jack A, Hamad MIK, Gonda S, Gralla S, Pahl S, Hollmann M, Wahle P (2018) Development of cortical pyramidal cell and interneuron dendrites: a role for kainate receptor subunits and NETO1. Mol Neurobiol. https://doi.org/10.1007/s12035-018-1414-0

45. Kelsch W, Li Z, Wieland S, Senkov O, Herb A, Göngrich C, Monyer H (2014) GluN2B-containing NMDA receptors promote glutamate synapse development in hippocampal interneurons. $\mathrm{J}$ Neurosci 34(48):16022-16030
46. Colin-LeBrun I, Ferrand N, Caillard O, Tosetti P, Ben-Ari Y, Gaiarsa J-L (2004) Spontaneous synaptic activity is required for the formation of functional GABAergic synapses in the developing rat hippocampus. J Physiol 559(Pt 1):129-139

47. Desai NS, Cudmore RH, Nelson SB, Turrigiano GG (2002) Critical periods for experience-dependent synaptic scaling in visual cortex. Nat Neurosci 5(8):783-789

48. Tien NW, Kerschensteiner D (2018) Homeostatic plasticity in neuronal development. Neural Dev 13(9):9. https://doi.org/10.1186/ s13064-018-0105-x

49. Sachidhanandam S, Blanchet C, Jeantet Y, Cho YH, Mulle C (2009) Kainate receptors act as conditional amplifiers of spike transmission at hippocampal mossy fibre synapses. J Neurosci 29(15):5000-5008

50. Fisher JL (2015) The auxiliary subunits Neto1 and Neto2 have distinct, subunit-dependent effects at recombinant GluK1- and GluK2containing kainate receptors. Neuropharmacology 99:471-480

51. Palacios-Filardo J, Aller MI, Lerma J (2016) Synaptic targeting of kainate receptors. Cereb Cortex 26(4):1464-1472

52. Crepel V, Mulle C (2015) Physiopathology of kainate receptors in epilepsy. Curr Opin Pharmacol 20:83-88

Publisher's Note Springer Nature remains neutral with regard to jurisdictional claims in published maps and institutional affiliations. 